\title{
Time-lapse lab-based X-ray nano-CT study of corrosion damage
}

DOI:

10.1111/jmi.12551

\section{Document Version}

Accepted author manuscript

Link to publication record in Manchester Research Explorer

\section{Citation for published version (APA):}

Lyon, S., Liu, Y., Zhou, X., Withers, P., Gholinia, A., \& Burnett, T. (2017). Time-lapse lab-based X-ray nano-CT study of corrosion damage. Journal of Microscopy, 267(1), 98-106. https://doi.org/10.1111/jmi.12551

\section{Published in:}

Journal of Microscopy

\section{Citing this paper}

Please note that where the full-text provided on Manchester Research Explorer is the Author Accepted Manuscript or Proof version this may differ from the final Published version. If citing, it is advised that you check and use the publisher's definitive version.

\section{General rights}

Copyright and moral rights for the publications made accessible in the Research Explorer are retained by the authors and/or other copyright owners and it is a condition of accessing publications that users recognise and abide by the legal requirements associated with these rights.

\section{Takedown policy}

If you believe that this document breaches copyright please refer to the University of Manchester's Takedown Procedures [http://man.ac.uk/04Y6Bo] or contact uml.scholarlycommunications@manchester.ac.uk providing relevant details, so we can investigate your claim.

\section{OPEN ACCESS}




\section{Microscopy}

\section{Time-lapse Lab-based X-ray nano-CT Study of Corrosion Damage}

\begin{tabular}{|r|l|}
\hline Journal: & Journal of Microscopy \\
\hline Manuscript ID & JMI-2016-0234.R1 \\
\hline Wiley - Manuscript type: & Original Article \\
\hline Domplete List of Authors: & $\begin{aligned} \text { Bradley, Robert; The Univesrity of Manchester, School of Materials ; } \\
\text { Geotek Ltd } \\
\text { Liu, Yanwen; The Univesrity of Manchester, School of Materials } \\
\text { Burnett, Timothy; The Univesrity of Manchester, School of Materials ; The } \\
\text { University of Manchester, Henry Moseley X-ray Imaging Facility } \\
\text { Zhou, Xiaorong; The Univesrity of Manchester, School of Materials } \\
\text { Lyon, Stuart; The Univesrity of Manchester, School of Materials } \\
\text { Withers, Philip; University of Manchester, School of Materials } \\
\text { Gholinia, Ali; University of Manchester, School of Materials } \\
\text { Hashimoto, Teruo; The Univesrity of Manchester, School of Materials } \\
\text { Graham, Derek; AkzoNobel } \\
\text { Gibbon, Simon; AkzoNobel } \\
\text { Hornberger, Benjamin; Carl Zeiss X-ray Microscopy }\end{aligned}$ \\
\hline Keywords: & $\begin{array}{l}\text { computed tomography, Zernike phase contrast, plasma focused ion beam } \\
\text { (PFIB), paint, primer coating }\end{array}$ \\
\hline
\end{tabular}




\title{
Time-lapse Lab-based X-ray nano-CT Study of Corrosion Damage
}

\author{
R.S. Bradley ${ }^{1 a^{*}}$, Y. Liü ${ }^{2^{*}}$, T.L. Burnett ${ }^{1,2}$, X. Zhou ${ }^{2}$, S.B. Lyon ${ }^{2}$, P.J. Withers ${ }^{1,2}$, A. Gholinia ${ }^{2}$, T. \\ Hashimoto $^{2}$, D. Graham ${ }^{3}$, S.R. Gibbon ${ }^{3}$, B. Hornberger ${ }^{4}$ \\ 1 Henry Moseley X-ray Imaging Facility, The University of Manchester, M13 9PL, UK \\ 2 School of Materials, The University of Manchester, M13 9PL, UK \\ 3 AkzoNobel, Stoneygate Lane, Felling, Gateshead, NE10 0JY, UK \\ 4 Carl Zeiss X-ray Microscopy, 4385 Hopyard Rd, Pleasanton, CA94588, U.S.A. \\ a Current address: Geotek Ltd, Daventry, Northamptonshire, NN11 8PB, UK; \\ *Rob.Bradley@geotek.co.uk; Tel: 00441327311666; Fax: 00441327311555 \\ *Yanwen.Liu@Manchester.ac.uk; Tel: 00441613065953; Fax: 00441613064865
}

\section{ABSTRACT}

An experimental protocol (workflow) has been developed for time-lapse x-ray nanotomography (nano-CT) imaging of environmentally-driven morphological changes to materials. Two case studies are presented. Firstly, the leaching of nanoparticle corrosion inhibitor pigment from a polymer coating was followed over 14 days, while in the second case the corrosion damage to an AA2099 aluminium alloy was imaged over 12 hours. The protocol includes several novel aspects relevant to nano-CT with the use of a combination of x-ray absorption and phase contrast data to provide enhanced morphological and composition information, and hence reveal the best information to provide new insights into the changes of different phases over time. For the pigmented polymer coating containing nominally strontium aluminium polyphosphate, the strontium-rich components within the materials are observed to leach extensively while the aluminium-rich components are more resistant to dissolution. In the case of AA2099 it is found that the initial grain boundary corrosion is driven by the presence of copper-rich phases and is then followed by the corrosion of grains of specific orientation.

Keywords: computed tomography, Zernike phase contrast; plasma focused ion beam (PFIB); paint; primer coating 


\section{INTRODUCTION}

Corrosion damage to metals typically proceeds over a wide range of spatial and temporal scales over which microstructure plays a key role (Liu, Zhou et al. 2007, Hashimoto, Zhou et al. 2010, Burnett, Kelley et al. 2016, Ma, Zhou et al. 2016, Zhang, Hashimoto et al. 2016). Furthermore polymer coatings containing corrosion inhibiting pigments are commonly applied for the corrosion protection of metals (Visser, Liu et al. 2015, Emad, Zhou et al. 2016, Liu, Visser et al. 2016, Liu, Zhou et al. 2016, Lyon, Bingham et al. 2016). Microstructural analysis and imaging is therefore critical to understanding the susceptibility of materials to corrosion damage as well as to understand how the leaching of inhibitive pigments from protective polymer coatings proceeds. Recent advances in 3-D imaging techniques based on electron microscopy, such as serial block face scanning electron microscopy (SBFSEM), employing sequential slicing of the materials with either a diamond knife (Hashimoto, Zhou et al. 2010, Hashimoto, Thompson et al. 2016) or an ion beam (Burnett, Kelley et al. 2016, Liu, Zhou et al. 2016) have enabled $5 \mathrm{~nm}$ resolution or better to be achieved over relatively large volumes (10's-100's $\mu \mathrm{m}$ cube). However, these techniques are destructive, and are therefore not amenable to time-lapse studies of dynamic processes occurring local to a given microstructural features. Xray micro tomography has been applied both at synchrotron sources (Babout, Marrow et al. 2006, Connolly, Horner et al. 2006) and using laboratory systems (Burnett, Holroyd et al. 2015) to study corrosion processes over time at the micron scale. Recently it has become possible to undertake time-lapse imaging of regions of interest around $50 \mu \mathrm{m}$ in dimension at resolutions of $\sim 50 \mathrm{~nm}$ or better using laboratory nano-CT systems (Patterson, Cordes et al. 2016). However, to date very 
little work (Krebs, Chirazi et al.) has exploited laboratory based x-ray nano-CT for studying the corrosion and protection of metallic materials. That is because the application of time-lapse $\mathrm{x}$-ray nano-CT is not straightforward in practice due to a number of experimental obstacles, including: sample preparation, data acquisition, and data processing.

The first challenge is to produce a sample of a suitable size for analysis by nano-CT. Nano-scale (sub-micron) x-ray imaging can be achieved through a variety of microscope designs (Withers 2007). For example, the full-field transmission x-ray microscope (TXM) is suitable for both synchrotron as well as laboratory $\mathrm{x}$-ray sources (Withers 2007) and has spatial resolutions down to $\sim 15 \mathrm{~nm}$ at the synchrotron and $\sim 50 \mathrm{~nm}$ in laboratory systems (Tkachuk, Duewer et al. 2007, Withers 2007). Given the size of current CCD cameras, the maximum field of view of a TXM is typically around $100 \mu \mathrm{m}$ for a $50 \mathrm{~nm}$ pixel size (Maire and Withers 2014). However, TXMs rely on Fresnel zone plates for beam focussing with characteristic radiation from either $\mathrm{Cr}(5.4 \mathrm{keV}), \mathrm{Cu}(8.04 \mathrm{keV})$ or $\mathrm{Mo}(17.3 \mathrm{keV})$ targets, limiting the penetration of $\mathrm{x}$-rays into metallic samples (i.e. from hundreds of microns for $\mathrm{Mg}$ to tens of microns for Fe). Preparation of suitable samples therefore requires specialised equipment in most cases. Laser cutting and focussed ion beam (FIB) milling have been successfully used (Michael 2011, Echlin, Mottura et al. 2012). However laser cutting typically introduces a narrow heat damaged zone (Echlin, Mottura et al. 2012). Furthermore, there are several parameters which need to be optimised to achieve successful cutting, including laser pulse duration and rate (Echlin, Mottura et al. 2012). FIB milling using gallium ions is suitable for a wide range of materials but is relatively slow, with preparation of a suitable nano- 
tomography sample taking more than 10 hours. Furthermore, there can be implantation of gallium within the materials, including along grain boundaries, which will influence corrosion processes especially of susceptible materials such as aluminium (Michael 2011). Recently plasma Xe+ FIB (PFIB) milling has been adapted for material science applications (Burnett, Kelley et al. 2016). In comparison to Ga+ FIB milling, it leads to reduced physical and chemical damage and achieves improved milling rates, typically $20-60 \times$ higher, making the preparation of the relatively large samples required for nano-CT practical (Burnett, Kelley et al. 2016). Although such equipment is costly, it produces minimal surface damage and can be used to extract specific volumes of interest, as identified by preceding microtomography.

With respect to experimental design, the choice of in-situ or ex-situ scanning also needs to be considered carefully. Corrosion can occur over a wide range of timescales, and for faster processes the sample should ideally be kept in the corrosive environment during scanning. In-situ scanning has been used for synchrotron x-ray tomography (Eckermann, Suter et al. 2008), providing data acquisition in minutes or even seconds. However, the design of suitable in-situ environmental chambers for x-ray nanoCT is particularly challenging, since: (1) it is necessary to be able to collect radiographs unhindered by the apparatus used to contain the desired sample environment over a wide range of rotation angles (to minimise artefacts in the volumetric data associated with limited angle scans); (2) apparatus also needs to be accommodated within a limited space within the sample stage area; (3) the limited penetration of 5-12 keV $\mathrm{x}$-rays through aqueous solution and containers for the fluid needs to be considered (e.g. the absorption length 
through water at $6 \mathrm{keV}$ is $\sim 40 \mu \mathrm{m})$. The alternative is to remove the sample from the corrosive environment periodically resulting in ex-situ scanning.

Synchrotron nano-CT typically takes $\sim 5-30$ minutes to record a single tomogram, and is therefore suitable for in-situ studies of dynamic processes which occur on times scales of 10 's of minutes or longer. Laboratory imaging takes considerably longer (typically 4-36 hours per tomogram) (Maire and Withers 2014) and is therefore more suited to ex-situ time-lapse corrosion studies. Both laboratory and synchrotron imaging can utilise $x$-ray phase contrast via the Zernike method in which a phase plate is introduced in the backfocal plane of the objective (Withers 2007) lens. X-ray phase contrast improves the visibility of low absorbing materials and can improve contrast between features having similar densities (Bradley and Withers 2016).

Analysis of the time-lapse 3-D volumetric data can reveal quantitative information on the rates and propagation of corrosion (Maire and Withers 2014). The analysis typically involves segmenting the features in the reconstructed data by, for example, applying greyscale thresholding. However, for meaningful conclusions to be drawn it is important to understand the variation between data sets recorded at the different time steps due to variability inherent in the tomographic process. Noise in the radiographs places a fundamental limit on the bias and variance of measurements made from the resulting volumetric data. Recently, Bradley adapted a statistical technique (SIMEX) to estimate these quantities from a single scan (Bradley 2016). Variations between data sets can also arise from changes in the x-ray source output and detector sensitivity over time. The effects of these 'systematic' variations can be 
reduced using local thresholding techniques for segmentation or by matching greyscale histograms of the volumes by applying a linear scaling such that the cumulative density functions are in good agreement (Lowe, Bradley et al. 2015). Alternatively, Bradley proposed an approach based on a 'volumetric standard' (Bradley 2016), which is either a region of the sample that does not vary between scans, or a separate object that is invariant and is included in the scan volume. The threshold used for segmentation is then adjusted so that the volume of a given feature in the volumetric standard is kept constant. Bradley demonstrated that with this approach that the systematic variation could almost all be eliminated, which is especially important for nano-CT imaging as it may be more practical to choose faster scans with consequently reduced signal to noise. In corrosion studies the loss of or gain of material (as a corrosion product) makes this step particularly important.

Here an experimental protocol (workflow) for time-lapse nano-CT imaging of corrosion processes has been developed, taking into account these challenges. This has been applied to imaging the corrosion damage in an AA2099 aluminium lithium alloy and the leaching of solid pigments from a polymer coating both as a result of exposure to $0.6 \mathrm{M}$ sodium chloride solution. In both cases, the nanoscale resolution achieved is critical in understanding of the mechanism of dissolution/inhibition by the polymer coating, and the propagation of corrosion in the alloy. Steel sheet used in domestic goods and architectural applications is almost invariably protected from corrosion damage by 'coil-coating' using polymer coatings containing corrosion inhibitive pigment (e.g. strontium aluminium polyphosphate) particles of dimension tens of nanometres to a few microns. Furthermore, the high-strength AA2099 aluminium alloy (Al-Cu-Li-Zn-Mg-Mn), used in aerospace applications, contains 
micro- and nano-scale constituent intermetallics, dispersoids and fine precipitates that are essential for its mechanical properties. This type of lithium-containing aluminium alloy is increasingly replacing traditional aluminium-copper alloys for aerospace applications in recent years due to its high strength and stiffness, lowest structural weight, and outstanding low temperature performance. The nanoscale resolutions achieved here provide critical information that enable correlation of the initiation and propagation of corrosion with the alloy microstructure.

\section{EXPERIMENTAL PROTOCOL}

A schematic workflow for time-lapse imaging of corrosion behaviour of the alloy and leaching of corrosion inhibitor from a primer coating is displayed in Fig. 1. A detailed explanation of each steps are given in the following sections.

\subsection{Sample preparation}

Primer coating study: A polyester model primer coating approximately $10 \times 10 \mathrm{~cm}$ and $14 \mu \mathrm{m}$ thick was formed onto PTFE film using a drawbar. It was then removed from the PTFE film and cut to $2 \times 2 \mathrm{~mm}$ with a surgical blade and attached to a polystyrene support with superglue. The sample was further cut to an approximately circular shape having a diameter around $65-70 \mu \mathrm{m}$. The primer coating is pigmented with an 'environmentally-friendly' corrosion inhibiting pigment: strontium aluminium polyphosphate (SAPP). Energy dispersive X-ray analysis (EDX) and X-ray diffraction analyses (XRD) showed that individual SAPP particles comprised one or more of: strontium aluminium polyphosphate enriched with strontium, strontium aluminium 
polyphosphate enriched with aluminium, strontium carbonate, strontium phosphate/polyphosphates, and aluminium phosphate/polyphosphate/hydrogen phosphate hydrate. Small amounts of zinc aluminium polyphosphate were also detected. Crystalline strontium carbonate and aluminium phosphate/polyphosphates were determined by electron backscattering diffraction in an FEI Nova NanoLab dual beam FIB-SEM microscope and high resolution TEM in Tecnai F30 transmission electron microscopy. Amorphous SAPP particles were also found. The SAPP has been categorised into two groups. The first group is named strontium-rich particles, including strontium carbonate, strontium polyphosphates, strontium aluminium polyphosphate enriched with strontium. The second group is named aluminium-rich particles, including aluminium phosphate/polyphosphate/hydrogen phosphate hydrate and strontium aluminium polyphosphate enriched with aluminium.

The coating was first imaged using nano-CT before being immersed in $0.6 \mathrm{M}$ sodium chloride solution for sequential periods of 1 day, 6 days, and 7 days, giving accumulated exposures of 1 day, 7 days and 14 days. X-ray nano-CT scans were taken after each immersion interval.

Aluminium alloy study: A metallic pillar, of about $34 \times 34 \times 70 \mu \mathrm{m}$, was prepared from the middle of an extruded stock section of AA 2099-T83 aluminium alloy from Airbus (1.62 wt. \% Li; 2.83 wt.\% Cu; 0.72 wt.\% Zn; 0.30 wt.\% Mg; 0.29 wt.\% Mn; 0.04 wt.\% Fe; 0.06 wt.\% K; 985 ppm Zr; 180 ppm Si; 1.5 ppm Ag; remainder, Al). The sample was cut into $2 \times 0.5 \times 10 \mathrm{~mm}$ diameter and ground into about $0.5 \mathrm{~mm}$ diameter tip. It was then prepared to its final dimensions using an FEI Helios plasma FIB using $\mathrm{Xe}+$ ions. The long axis of the pillar is parallel to the extrusion direction. The sample was 
repeatedly returned to a $0.6 \mathrm{M}$ sodium chloride solution for time intervals of $0.5,1.5$, and $10 \mathrm{~h}$ giving accumulated immersion exposures of $0,0.5,2$, and $12 \mathrm{~h}$. X-ray nano-CT scans were taken before immersion and after each immersion period.

\subsection{X-ray nano-CT scanning}

X-ray nano-CT was carried out using a Zeiss Xradia 810 Ultra x-ray microscope (Carl Zeiss X-ray Microscopy, Pleasanton, California, U.S.A.). An absorption contrast and a phase contrast scan were recorded before immersion and after each immersion interval for both samples. The samples were kept secure within the same sample holder for scanning and immersion, to ensure that the orientation of the samples was the same for all scans. Care was taken to ensure the samples were in approximately the same position in the field of view for all scans. Scan conditions for each scan are shown in Table 1.

\subsection{Data analysis}

Volumetric data was reconstructed with a voxel size of $63.6 \mathrm{~nm}$ for phase contrast scans and $127.7 \mathrm{~nm}$ for absorption contrast scans using filtered back projection via the TomoTools interface (Bradley 2015) to the ASTRA toolbox (van Aarle, Palenstijn et al. 2015) within Matlab (Mathworks, Natick, MA). Any sample drift during the scan was corrected by the approach of Myers et al. (Myers, Kingston et al. 2011) in which the radiographs are registered to those of a 'short scan' having a reduced number of radiographs (in the range 11 to 25 ).

After reconstruction, the image volumes were spatially registered together, using the registration tools in Avizo 9.0 (FEI VSG, Burlington, MA). This is a key step when 
tracking changes in specific features between time steps. For this, the initial phase contrast scan of each sample, taken before immersion, was used as a reference. Subsequent phase contrast tomograms were aligned to this reference tomogram. The absorption contrast data was then aligned to the phase contrast data at each immersion interval. The grey levels in each scan were also matched by scaling the data such that the cumulative density functions (cumulative probability density for the greyscale data, CDF) were in good agreement with that of the first scan, which also adds confidence in tracking changes and following dynamic events. This involved scaling the data using a second order polynomial until the CDF was in good agreement with that of the initial scan. This optimisation was carried out automatically using the Levenberg-Marquardt algorithm in Matlab, and was carried out separately for the absorption and phase contrast data. Noise in the data sets was then reduced by applying guided image filtering on each slice (D'Errico 2005) using the 'imguidedfilter' Matlab function.

The particles in the coating were segmented for further analysis using a greyscale threshold applied to the phase contrast data, which provides clear definition of the particle boundaries. Small isolated regions in the segmentation arising from noise in the scan data were then removed. The particle sizes and spacings were measured by calculating the local thickness (Hildebrand and Rüegsegger 1997) within the particle and matrix segmentations respectively. The strontium-rich and aluminium rich components were then separated by using the absorption contrast data. The absorption contrast data provides great contrast between different particle types but poorer boundary definition in comparison to phase contrast. First, the absorption contrast data were up-sampled to the same voxel size as the phase contrast data, 
and then a grey threshold level was set so as to select only the strontium-rich particles. Here the phase contrast data was used to create a binary image highlighting all particles (i.e. a label-field). A Matlab code developed in-house was then used to compare each voxel identified as a particle to the corresponding voxel in the absorption data to identify what type of particle it was. Particle voxels (as labelled using the phase contrast data) were then labelled as strontium-rich if at least $50 \%$ of the voxels in a $9 \times 9 \times 9$ window, centred on each particle voxel, were labelled as strontium rich in the absorption contrast data (Hildebrand and Rüegsegger 1997).

\section{RESULTS}

\subsection{Absorption vs phase contrast imaging}

A 3D x-ray nano-CT absorption image of SAPP pigmented polymer, around $14 \mu \mathrm{m}$ thick on average, is displayed in Fig. 2. The particles appear with varying brightness dependent upon their average atomic number compared with the polymer binder, which is of dark appearance. Briefly, the brighter particles are strontium-rich particles, of strontium carbonate, strontium phosphinate/polyphosphates, and strontium aluminium polyphosphate enriched with strontium. Zinc aluminium polyphosphates also showed brighter appearance. The less bright/grey particles are mainly strontium aluminium polyphosphate enriched with aluminium, aluminium phosphate/polyphosphate/hydrogen phosphate hydrate.

A comparison between absorption and Zernike phase contrast scans can be seen in Fig. 3. It is evident that the Zernike phase contrast provides clearer morphologic details while absorption contrast reveals significant composition information, i.e. 
atomic number contrast between different elements in the inhibitor (Fig. 3). This is because phase contrast enhances edges, especially between low contrast difference materials. The combination of absorption and phase contrast scans can therefore yield complementary information and facilitate segmentation of the different phases with good boundary definition.

\subsection{Exemplar 1: Time-lapse study of the leaching of inhibitor from a primer coating}

The evolution of the polymer coating containing SAPP particles during immersion in 0.6 M sodium chloride solution is displayed in a virtual cross section in Fig. 4. Two regions are examined in detail as shown in the figure. The first is associated with strontium-rich particles which have a brighter appearance (greater x-ray attenuation) and a median size (local thickness) of $1.3 \mu \mathrm{m}$ while the second is associated with aluminium-rich particles, which are less attenuating and smaller with a median size of $0.5 \mu \mathrm{m}$. It is evident that material is released from the coating over the course of the experiment, as illustrated by the (strontium-rich) particles marked with the oval shape, leaving cavities at their original locations. Importantly this is the first direct evidence for pathways allowing ingress and egress of ionised species within a nanocomposite structure that comprises a polymer coating. The majority of the aluminium-rich particles were relatively stable although some dissolution was observed, especially after 7 days of immersion (see the regions with a square mark). Interestingly, such dissociation did not result in rounded cavities. Instead, they appeared angular, possibly associated with the original crystalline structure of the particles. The 3D dissolution of a relatively large strontium-rich component particle is 
shown in the time-lapse sequence in Fig. 5, which reveals that after 14 days of immersion, most of it had been dissolved.

While 3D imaging with time can provide direct evidence for the different leaching behaviours of the different components within the SAPP particle conglomerates, the data also supply non-destructive quantification of volumetric changes within the polymer coating. Table 2 presents the changes of the total pigment volume and the relative ratios of strontium-rich particles to the aluminium-rich particles. These results are consistent with the similar study of leaching behaviour of SAPP in the coating, which showed a much higher dissolution rate of strontium-rich particles comparing with aluminium-rich particles $(9,10)$. The bias and variance of the nano-CT measurements were estimated using the SIMEX technique [24]. It was found that noise in the scan data led to the volume fractions being underestimated (negatively biased) by a relatively small amount $(<0.5 \%)$, while the standard deviations were estimated to be much smaller at $<0.01 \%$. The underestimation is likely to be due to the step taken in the segmentation procedure to remove small isolated regions, the majority of which represent noise in the scan data. Retaining these regions would have led to a larger overestimation of $\sim 1.5 \%$. Further variation arising from systematic changes in greyscales between data sets (not fully accounted for by the rescaling step) and the finite precision of the 3-D registration were estimated to be $\sim 0.3 \%$ for the measured volumes fractions. Nevertheless, these findings demonstrate that nano-CT can provide sufficiently high quality, relatively low-noise data to enable quantitative measurements to be reliably compared between time periods. 


\subsection{Exemplar 2: Time-lapse imaging of corrosion damage in AA2099 alloy}

The same time-lapse approach was used to study the corrosion development in AA2099 aluminium alloy as a function of immersion time in a $0.6 \mathrm{M}$ sodium chloride solution. Virtual slices, from the Zernike phase contrast scans, of the alloy before and after immersion are shown in Figs. 6 a-d. The initial scan before immersion (Fig. 6 a) revealed microstructural features in the alloy, including constituent intermetallics (indicated by oval regions), dispersoids and bands of grain boundary precipitates (indicated by arrows). The brighter appearance of the intermetallics, dispersoids and grain boundary precipitates suggested that they contained elements with higher atomic number than the aluminium matrix. EDX analyses confirmed that the intermetallics present in the alloy contain $\mathrm{Al}, \mathrm{Fe}, \mathrm{Mn}$ and $\mathrm{Cu}$. The rod-like particles (indicated by the square mark in Fig. 6 a) contained aluminium, iron and manganese. The irregularly shaped particles (indicated by the oval mark in Fig. 6 a) are likely to contain aluminium, copper, iron and manganese (Ma 2011). The grains are elongated in the extrusion direction. Higher copper contents were detected at the grain boundaries, which are related to the precipitation of $T_{1}\left(\mathrm{Al}_{2} \mathrm{CuLi}\right), \mathrm{T}_{2}\left(\mathrm{Al}_{6} \mathrm{CuLi}_{3}\right)$ and $T_{B}$ phases $\left(\mathrm{Al}_{7} \mathrm{Cu}_{4} \mathrm{Li}\right)$ (Ma 2011, Ma, Zhou et al. 2011) at high angle grain boundaries. Fine precipitates $\delta^{\prime}\left(\mathrm{Al}_{3} \mathrm{Li}\right), \mathrm{T}_{1}\left(\mathrm{Al}_{2} \mathrm{CuLi}\right), \theta^{\prime}\left(\mathrm{Al}_{2} \mathrm{Cu}\right)$ are found as well in the matrix and grain boundaries by TEM examination (Ma, Zhou et al. 2011). A detailed characterisation of the grains and grain boundaries has been carried out (Liu et al 2016). The corrosion behaviour and its relation to the microstructure in this study will be reported in a separate paper by a correlative imaging scheme bringing together x-ray nano-CT, high resolution transmission electron microscopy and scanning electron microscopy (Liu et al 2016). 
Here we compare the x-ray nano-CT before and after immersion in $0.6 \mathrm{M} \mathrm{NaCl}$ for 0.5 and $2 \mathrm{~h}$, it is evident that active corrosion was initiated at the regions of grain boundaries. The intergranular corrosion has propagated inwards along the grain boundaries over time from the surface of the alloy (Fig. 6 b) into the alloy matrix (Fig. $6 \mathrm{~b}-\mathrm{c})$. After prolonged immersion (12 h) corrosion developed within the interior of selected grains. There is considerable controversy in the literature regarding the initiation and propagation of corrosion in AA2099 aluminium alloy. Thus, either preferential corrosion of active T1 phase in the grain boundaries (Buchheit Jr, Moran et al. 1990), or formation of copper depleted zones in the grain boundaries (Kumai, Kusinski et al. 1989), results in active grain boundary corrosion. On the other hand, Ma et al. (Ma, Zhou et al. 2016) has found grain orientation can directly indicate corrosion susceptibility of the grain/subgrain in AA 2099-T83 alloy, with localised corrosion preferentially occurring on grains which experienced more plastic deformation. Here, direct evidence shows that the corrosion of grain boundaries have dominated the initial stage of the corrosion of 0.5 and $2 \mathrm{~h}$ whereas after prolonged immersion time, selective corrosion of grains was observed.

A time-lapse representation of the corrosion process of the alloy is shown in Fig. 7. The 3D representation of corrosion behaviour, and its relation with the microstructure of the alloy, is clearly revealed with the corrosion front inside the alloy highlighted at different time scales by colour coding.

\section{DISCUSSIONS AND CONCLUSIONS}

The case studies presented in this paper suggest that $\mathrm{x}$-ray nano-CT is a powerful tool for 3D time-lapse imaging of environmental damage to materials. However, care 
needs to be followed to achieve high quality data and reliable quantification of the changes at sufficiently high resolution. Current lab-based nano-CT systems provide regions of interest of the order of $60 \mu \mathrm{m}$ at $150 \mathrm{~nm}$ resolution and $15 \mu \mathrm{m}$ at $50 \mathrm{~nm}$ resolution (Tkachuk, Duewer et al. 2007, Withers 2007). The former is sufficient for the observation of protective coatings and corrosion of light alloys and, where necessary, larger volumes may be interrogated by image stitching. Zernike phase contrast provides nanoscale morphological information of the materials, while the absorption contrast scans supply compositional information for individual element quantification. Absorption contrast is dependent on atomic number and density, so addition techniques (e,g, EDX) must be employed for precise element identification. However, since nanoCT is non-destructive, the changes in the materials can be observed directly and repeatedly to form a time-lapse sequence. Acquisition times for laboratory systems are usually 6-24 hours, which is compatible with long timescale events that occur over weeks and can be imaged in operando. For shorter time scale events one should interrupt or 'freeze' the behaviour to enable ex-stu studies such as the one presented here to be carried out. Alternatively, synchrotron imaging can capture events occurring in-situ over minutes.

In the case of leaching of solid phases from polymers, here the dissolution of strontium aluminium polyphosphate was related to the relative amount of strontium in the conglomerate particles. Particles containing higher amounts of strontium, showed enhanced contrast (brighter grey-level) in both phase contrast and absorption contrast scans, although the contrast difference was much greater in the absorption contrast data. The phase contrast scans provided clear definition of the particle boundaries and revealed the cavities left by the leaching of strontium-rich 
particles. The combination of phase and absorption contrast during segmentation then facilitated the automatic identification of these particles for quantification. The complete leaching of some smaller bright particles at the surface region was observed to occur within one day (Fig 4b). More importantly, the leaching behaviour of aluminium-rich SAPP in the primer coating has been clearly observed with this technique for the first time. Considering the development of corrosion in AA2099 aluminium alloy, it was found that corrosion initiated at, and propagated along, the grain boundaries of the alloy while corrosion in the grains was only observed after prolonged immersion of $12 \mathrm{~h}$ (Fig 6). Electron backscatter diffraction (EBSD) mapping was used to determine the influence of grain boundary misorientation on corrosion propagation along grain boundaries, which revealed that corrosion preferentially propagates along high angle grain boundaries (Liu, Y., et al. 2016). Finally the combination of Zernike phase contrast and absorption contrast scans used here can provide complementary information across a wide range of studies.

\section{ACKNOWLEDGMENTS}

The authors would like to acknowledge EPSRC funding for the Henry Moseley X-ray Imaging Facility under grants EP/M010619, EP/K004530, EP/F007906, EP/F028431. RSB is grateful to Zeiss for funding of his fellowship and $\mathrm{YL}$ to AkzoNobel for her fellowship.

\section{REFERENCES}


Babout, L., et al. (2006). "X-ray microtomographic observation of intergranular stress corrosion cracking in sensitised austenitic stainless steel." Materials science and technology 22(9): 1068-1075.

Bradley, R. S. (2016). "Estimation of bias and variance of measurements made from tomography scans." Measurement Science and Technology 27(9): 095402.

Bradley, R. S. and P. J. Withers (2016). "Correlative multiscale tomography of biological materials." MRS Bulletin 41(7): 549-556.

Bradley, R. S. (2016). " TomoTools. Github". https://github.com/rsbradley/tomotools.

Buchheit Jr, R., et al. (1990). "Localized corrosion behavior of alloy 2090-the role of microstructural heterogeneity." Corrosion 46(8): 610-617.

Burnett, T., et al. (2016). "Large volume serial section tomography by Xe Plasma FIB dual beam microscopy." Ultramicroscopy 161: 119-129.

Burnett, T. L., et al. (2015). "The role of crack branching in stress corrosion cracking of aluminium alloys." Corrosion Reviews 33(6): 443-454.

Connolly, B., et al. (2006). "X-ray microtomography studies of localised corrosion and transitions to stress corrosion cracking." Materials science and technology 22(9): 1076-1085. 
D’Errico, J. (2005). "Surface fitting using gridfit." MATLAB central file exchange 643. http://www.mathworks.com/matlabcentral/fileexchange/8998. Accessed June 2016

Echlin, M. P., et al. (2012). "A new TriBeam system for three-dimensional multimodal materials analysis." Review of Scientific Instruments 83(2): 023701.

Eckermann, F., et al. (2008). "In situ monitoring of corrosion processes within the bulk of AIMgSi alloys using X-ray microtomography." Corrosion Science 50(12): 3455-3466.

Emad, S. G. R., et al. (2016). "Influence of volume concentration of active inhibitor on microstructure and leaching behaviour of a model primer." Progress in Organic Coatings.

DOI:http://dx.doi.org/10.1016/j.porgcoat.2016.04.039.

Hashimoto, T., et al. (2016). "3D imaging by serial block face scanning electron microscopy for materials science using ultramicrotomy." Ultramicroscopy 163: 6-18.

Hashimoto, T., et al. (2010). "Nanotomography for understanding materials degradation." Scripta Materialia 63(8): 835-838.

Hildebrand, T. and P. Rüegsegger (1997). "A new method for the model-independent assessment of thickness in three-dimensional images." Journal of microscopy 185(1): $67-75$. 
Krebs, H., et al. "Time-lapse Correlative 3D Imaging Applied to the Corrosion Study of AZ31 Mg Alloy in a Saline Environment." in FiMPART'152015: Hyderabad, India.

Kumai, C., et al. (1989). "Influence of aging at $200 \mathrm{C}$ on the corrosion resistance of Al-Li and Al-Li-Cu alloys." Corrosion 45(4): 294-302.

Liu, Y., et al. (2016). "Protective film formation on AA2024-T3 aluminum alloy by leaching of lithium carbonate from an organic coating." Journal of The Electrochemical Society 163(3): C45-C53.

Liu, Y., et al. (2016). "An organic coating pigmented with strontium aluminium polyphosphate for corrosion protection of zinc alloy coated steel." Progress in Organic Coatings.

DOI: http://dx.doi.org/10.1016/j.porgcoat.2016.02.020

Liu, Y., et al. (2007). "Precipitation in an AA6111 aluminium alloy and cosmetic corrosion." Acta materialia 55(1): 353-360.

Liu, Y., et al. (2016). Mechanism of corrosion of AA2099 aluminium alloy explored by Time-lapse X-ray nano-CT, to be submitted

Lowe, T., et al. (2015). "Microstructural analysis of TRISO particles using multi-scale X-ray computed tomography." Journal of Nuclear Materials 461: 29-36. 
Lyon, S., et al. (2016). "Advances in corrosion protection by organic coatings: What we know and what we would like to know." Progress in Organic Coatings.

DOI: http://dx.doi.org/10.1016/j.porgcoat.2016.04.030

Ma, Y. (2011). "Effect of microstructure on corrosion resistance and anodising behaviour of AA 2099-T8 aluminium alloy.",Ph.D thesis, The University of Manchester

Ma, Y., et al. (2016). "Localised corrosion in AA 2099-T83 aluminium-lithium alloy: The role of grain orientation." Corrosion Science 107: 41-48.

Ma, Y., et al. (2011). "Distribution of intermetallics in an AA 2099-T8 aluminium alloy extrusion." Materials chemistry and physics 126(1): 46-53.

Maire, E. and P. Withers (2014). "Quantitative X-ray tomography." International materials reviews 59(1): 1-43.

Michael, J. R. (2011). "Focused ion beam induced microstructural alterations: Texture development, grain growth, and intermetallic formation." Microscopy and Microanalysis 17(03): 386-397.

Myers, G. R., et al. (2011). "Extending reference scan drift correction to highmagnification high-cone-angle tomography." Optics letters 36(24): 4809-4811. 
Patterson, B., et al. (2016). "In Situ Laboratory-Based Transmission X-Ray

Microscopy and Tomography of Material Deformation at the Nanoscale."

Experimental Mechanics 56(9): 1585-1597.

Tkachuk, A., et al. (2007). "X-ray computed tomography in Zernike phase contrast mode at $8 \mathrm{keV}$ with 50-nm resolution using Cu rotating anode X-ray source." Zeitschrift für Kristallographie 222(11/2007): 650-655.

van Aarle, W., et al. (2015). "The ASTRA Toolbox: A platform for advanced algorithm development in electron tomography." Ultramicroscopy 157: 35-47.

Visser, P., et al. (2015). "The corrosion protection of AA2024-T3 aluminium alloy by leaching of lithium-containing salts from organic coatings." Faraday discussions 180: 511-526.

Withers, P. J. (2007). "X-ray nanotomography." Materials today 10(12): 26-34.

Zhang, X., et al. (2016). "Investigation of the de-alloying behaviour of $\theta$-phase (Al 2 Cu) in AA2024-T351 aluminium alloy." Corrosion Science 108: 85-93.

\section{Figure captions:}


Fig. 1: A workflow diagram used for the time-lapse x-ray nano-CT study of leaching inhibitors and corrosion of AA2099 aluminium alloy, revealing each steps in data acquisition and processing.

Fig. 2: A 3D x-ray nano-CT absorption image of the SAPP pigmented primer coating (14 micron thick on average).

Fig. 3: A comparison between absorption (top part) and Zernike phase contrast scans (bottom part), which shows that the Zernike phase contrast scan reveals the morphology on greater detail while the absorption contrast reveals significant composition information, i.e. atomic contrast from different elements in the inhibitor.

Fig. 4: The evolution of the organic primer coating pigmented with SAPP corrosion inhibitor over the course of the immersive treatment days in sodium chloride solution: (a) before immersion; (b) after immersion for 1 day; (c) after immersion for 7 days; (d) after immersion for 14 days. The oval region highlights the dissolution of strontium-rich particles while the square region highlighted the activity of aluminiumrich particles.

Fig. 5: A 3D representation of the dissolution of SAPP particles in the primer coating after immersion in $0.6 \mathrm{M} \mathrm{NaCl}$ for the testing period: (a) before immersion; (b) after immersion for 1 day; (c) after immersion for 7 days; (d) after immersion for 14 days. 
Fig. 6: Virtual cross-sections of Zernike phase contrast tomographic images of the alloy before (a) and after immersion in sodium chloride solution for $0.5 \mathrm{~h}$ (b), $2 \mathrm{~h}$ (c) and $12 \mathrm{~h}(\mathrm{~d})$.

Fig. 7: A 3 D representation of the corrosion process of the alloy, with corrosion developed in 0.5 (blue), 2 (green), and $12 \mathrm{~h}$ (red) shown by the artificial colours.

Tables

Table 1: Nano CT scan settings for the two case studies

\begin{tabular}{|l|c|c|c|}
\hline \multirow{3}{*}{ Sample } & Setting & Phase contrast & $\begin{array}{l}\text { Absorption } \\
\text { contrast }\end{array}$ \\
\hline \multirow{3}{*}{ Coating } & Zone plate resolution $(\mathrm{nm})$ & 150 & 150 \\
\cline { 2 - 4 } & Camera binning $^{*}$ & 1 & 2 \\
\cline { 2 - 4 } & Exposure time (s) & 60 & 14 \\
\cline { 2 - 4 } & Number of radiographs & 721 & 901 \\
\hline \multirow{3}{*}{ AA2099 } & Zone plate resolution (nm) & 150 & 150 \\
\cline { 2 - 4 } & Camera binning & 1 & 2 \\
\cline { 2 - 4 } & Exposure time (s) & 120 & 15 \\
\cline { 2 - 4 } & Number of radiographs & 721 & 901 \\
\hline
\end{tabular}

* During readout camera pixels are summed to give a reduced number of image pixel. Binning 2 corresponds to $2 x 2$ pixels being summed to give 1 corresponding image pixel.

Table 2: Volume and Sr rich:Al rich inhibitor particle volume ratio changes for the SAPP after immersion in $0.6 \mathrm{M} \mathrm{NaCl}$ for 14 days

\begin{tabular}{|c|c|c|}
\hline Time & $\begin{array}{c}\text { Total volume fraction of } \\
\text { the inhibitor particles in } \\
\text { the primer coating } \\
\text { matrix (\%) }\end{array}$ & $\begin{array}{c}\text { Sr-rich: Al-rich } \\
\text { (Volume ratio) }\end{array}$ \\
\hline $\begin{array}{c}\text { Before } \\
\text { immersion }\end{array}$ & 20.3 & $15: 85$ \\
\hline After 1 day & 19.4 & $14: 86$. \\
\hline After 7 days & 18.9 & $9: 91$ \\
\hline After 14 days & 17.9 & $6: 94$ \\
\hline
\end{tabular}




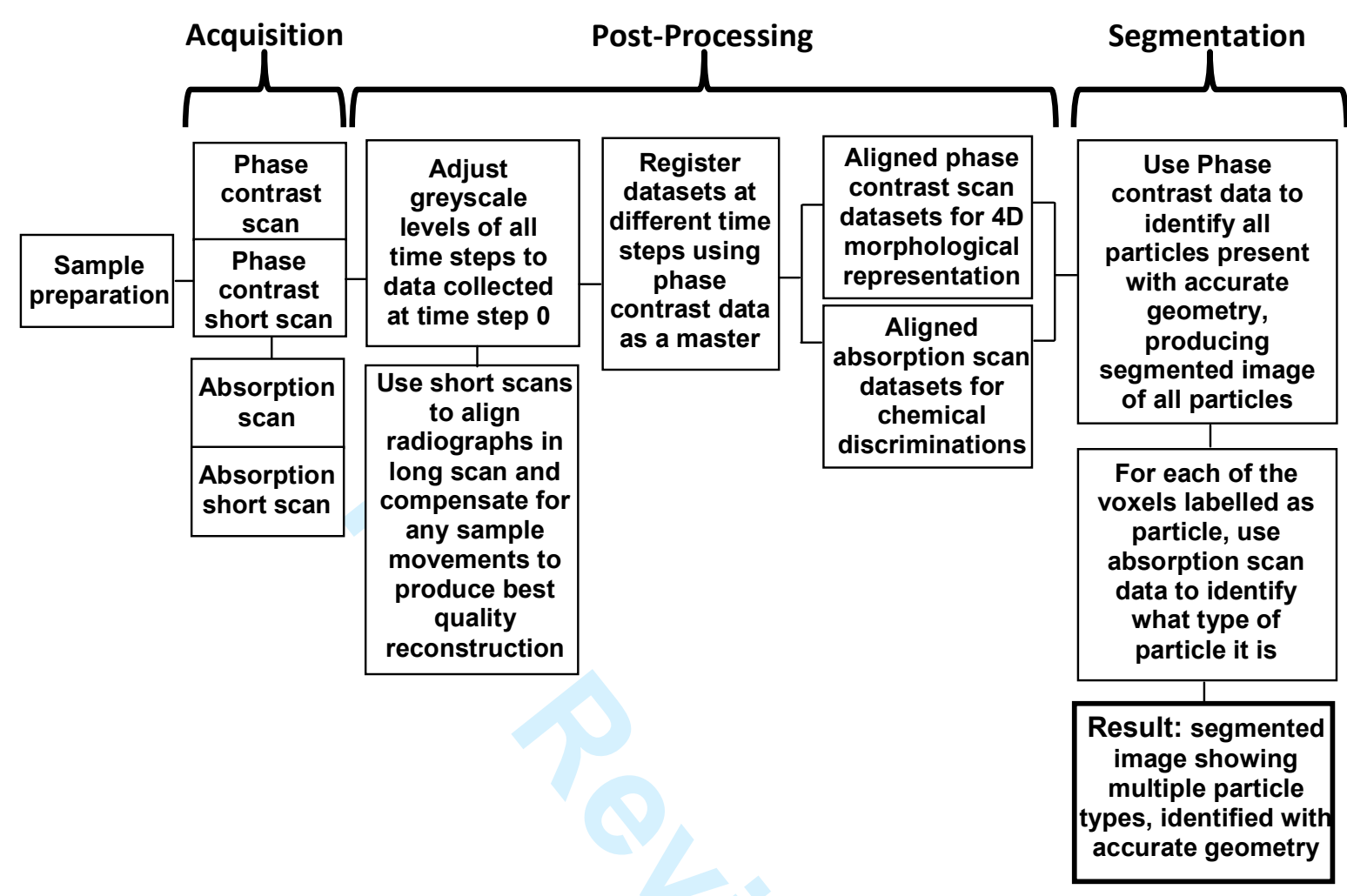

Fig. 1: A workflow diagram used for the time-lapse x-ray nano-CT study of leaching

inhibitors and corrosion of AA2099 aluminium alloy, revealing each steps in data acquisition and processing. 


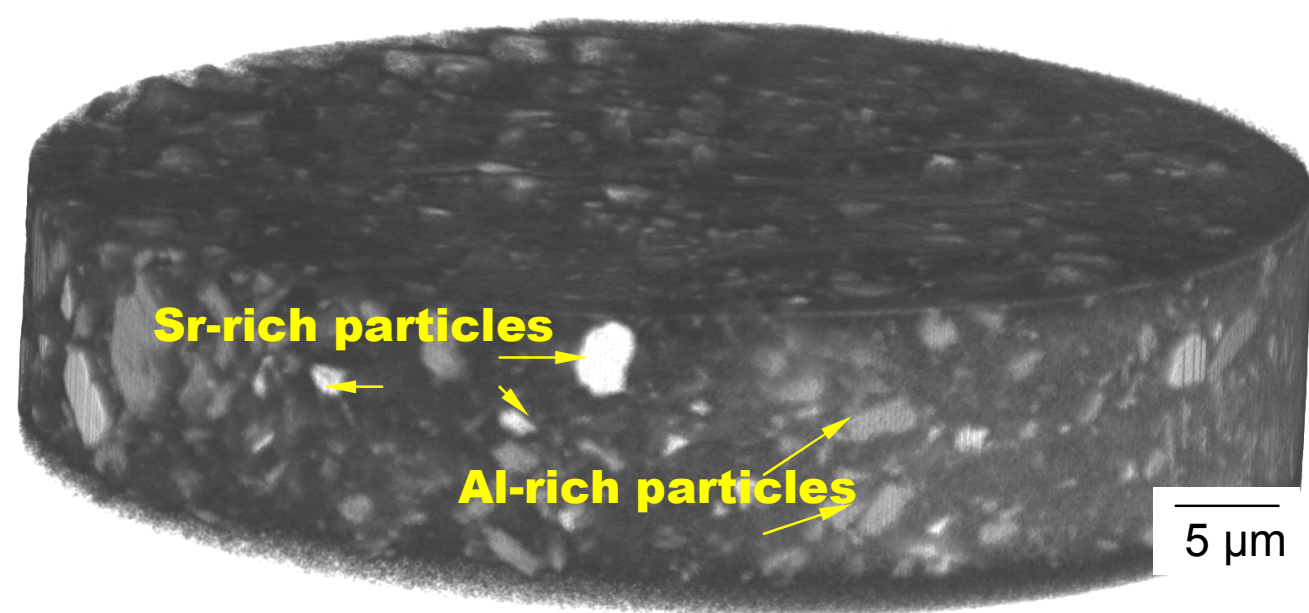

Fig. 2: A 3D x-ray nano-CT absorption image of the SAPP pigmented primer coating (14 micron thick on average). 


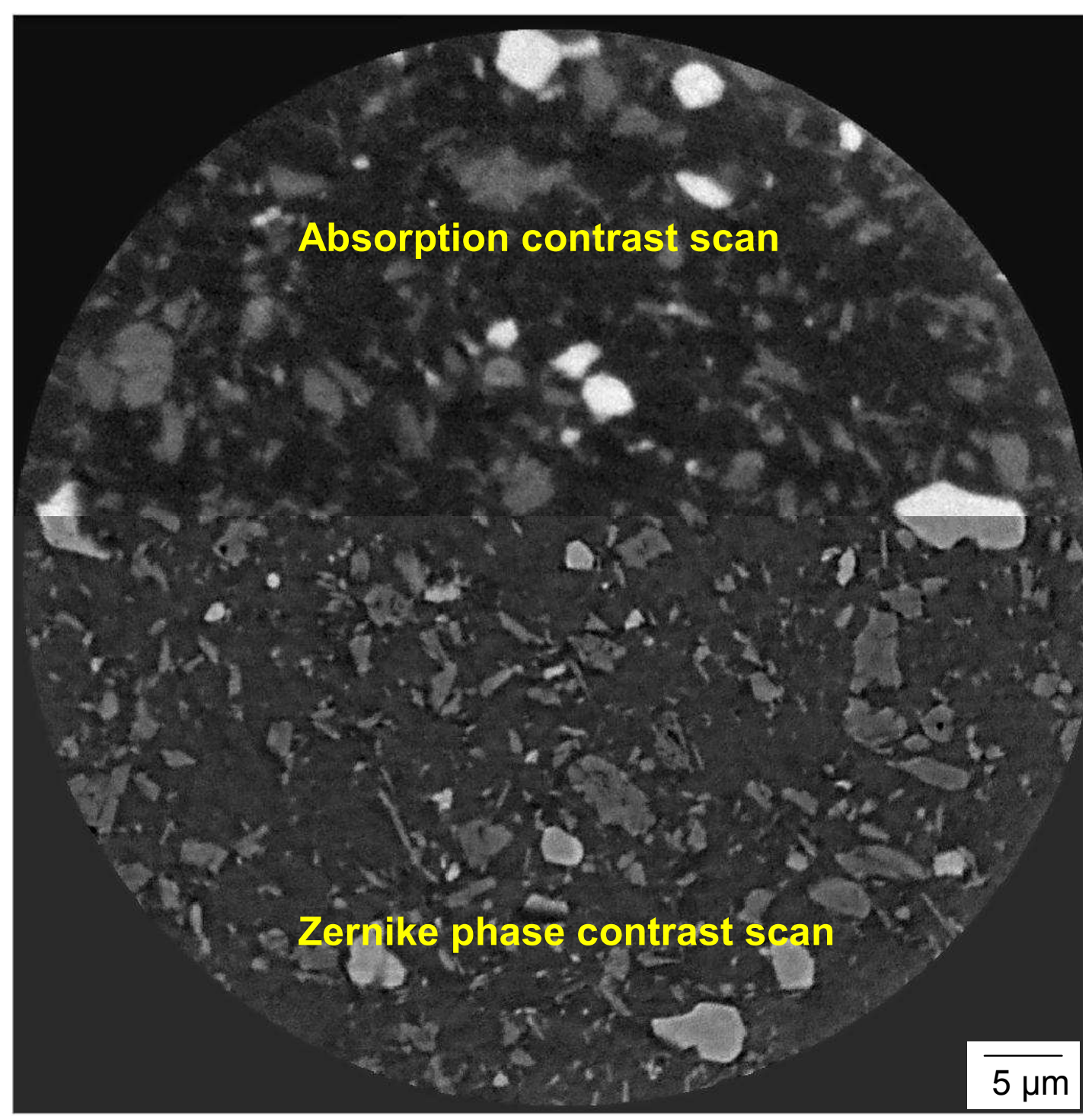

Fig. 3: A comparison between absorption (top part) and Zernike phase contrast scans (bottom part), which shows that the Zernike phase contrast scan reveals the morphology on greater detail while the absorption contrast reveals significant composition information, i.e. atomic contrast from different elements in the inhibitor. 

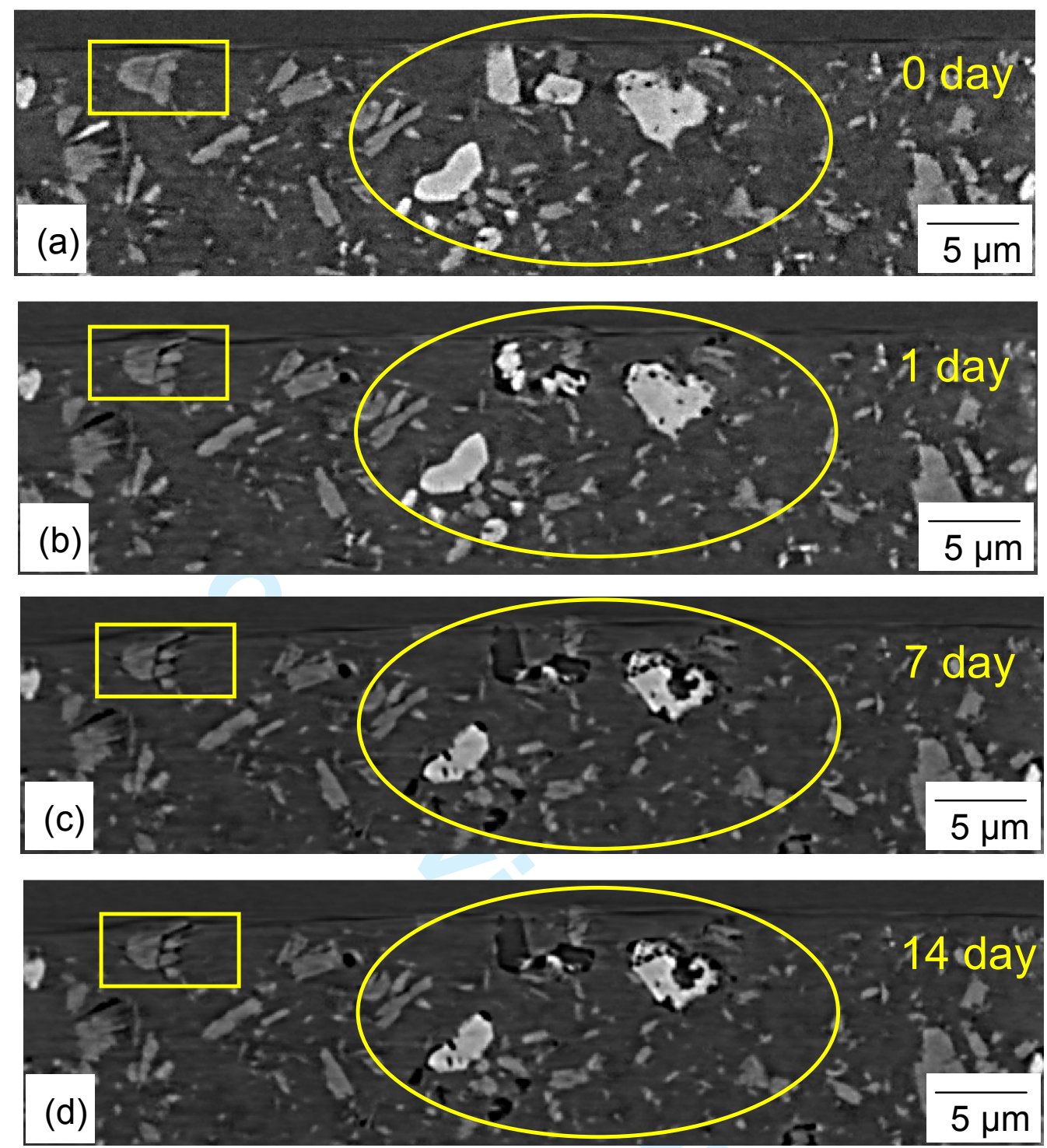

Fig. 4: The evolution of the organic primer coating pigmented with SAPP corrosion inhibitor over the course of the immersive treatment days in sodium chloride solution: (a) before immersion; (b) after immersion for 1 day; (c) after immersion for 7 days; (d) after immersion for 14 days. The oval region highlights the dissolution of strontium-rich particles while the square region highlighted the activity of aluminium-rich particles. 

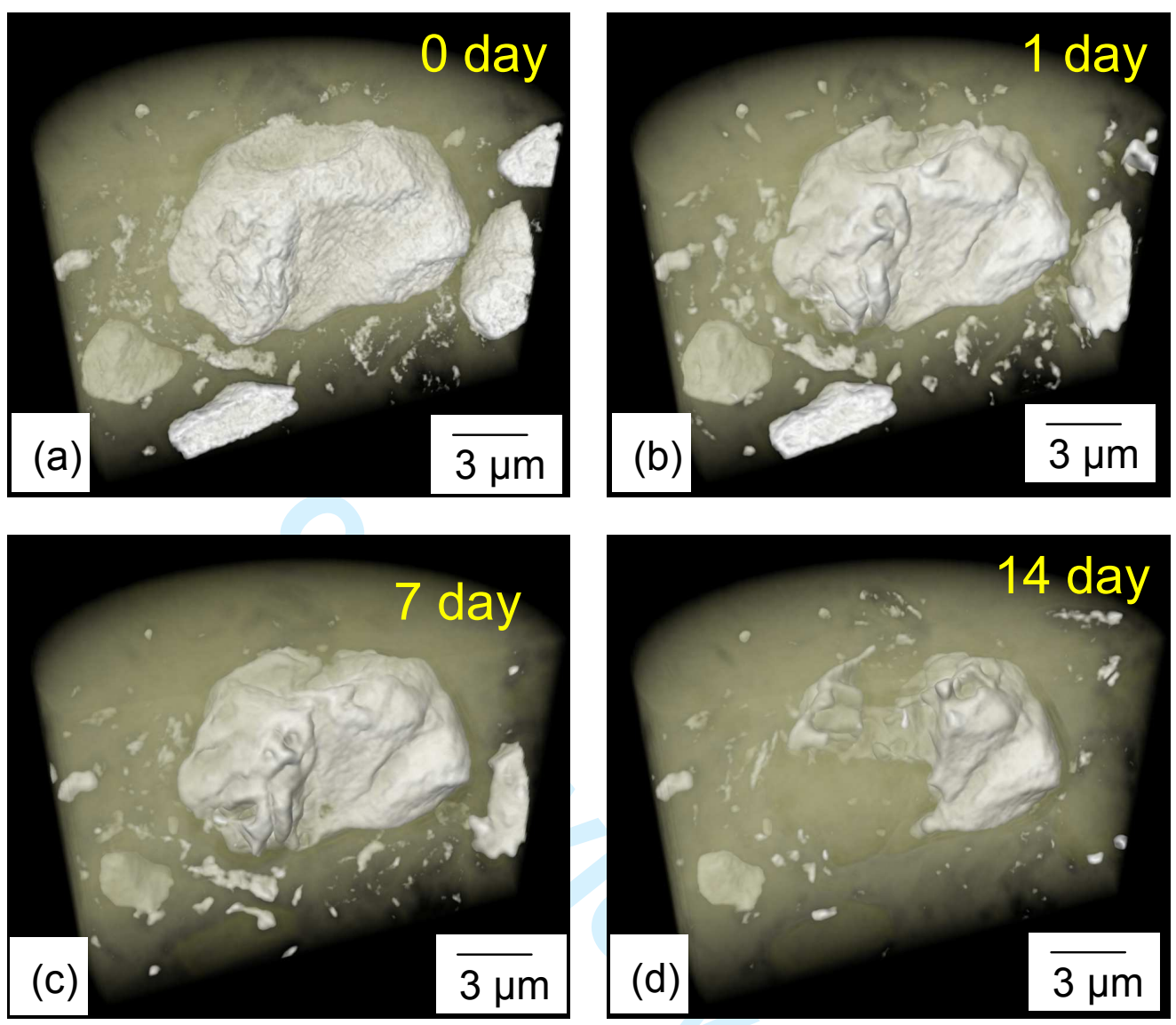

Fig. 5: A 3D representation of the dissolution of SAPP particles in the primer coating after immersion in $0.6 \mathrm{M} \mathrm{NaCl}$ for the testing period: (a) before immersion; (b) after immersion for 1 day; (c) after immersion for 7 days; (d) after immersion for 14 days. 

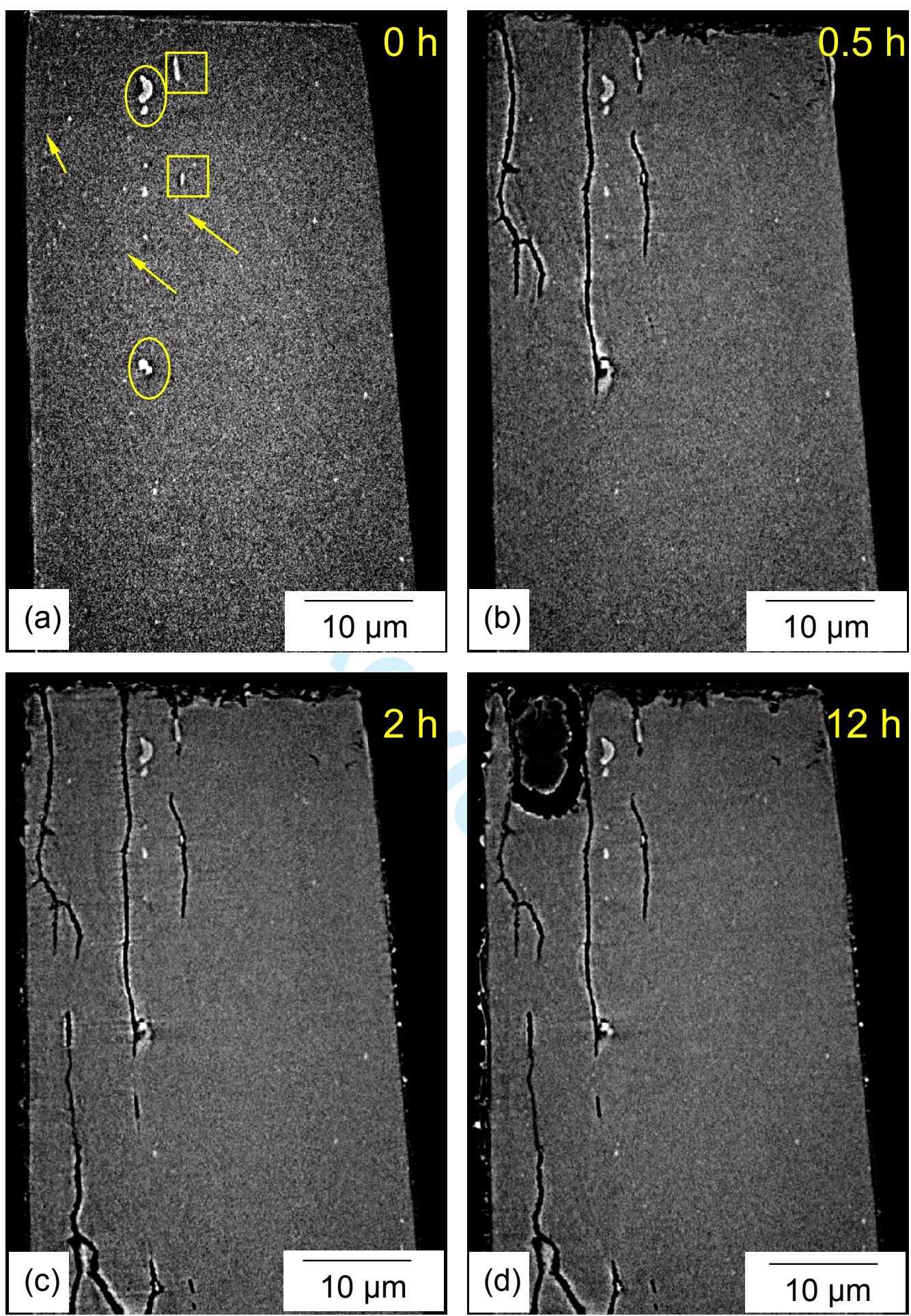

Fig. 6: Virtual cross-sections of Zernike phase contrast tomographic images of the alloy before (a) and after immersion in sodium chloride solution for $0.5 \mathrm{~h} \mathrm{(b),} 2 \mathrm{~h} \mathrm{(c)} \mathrm{and} 12 \mathrm{~h}$ (d). 


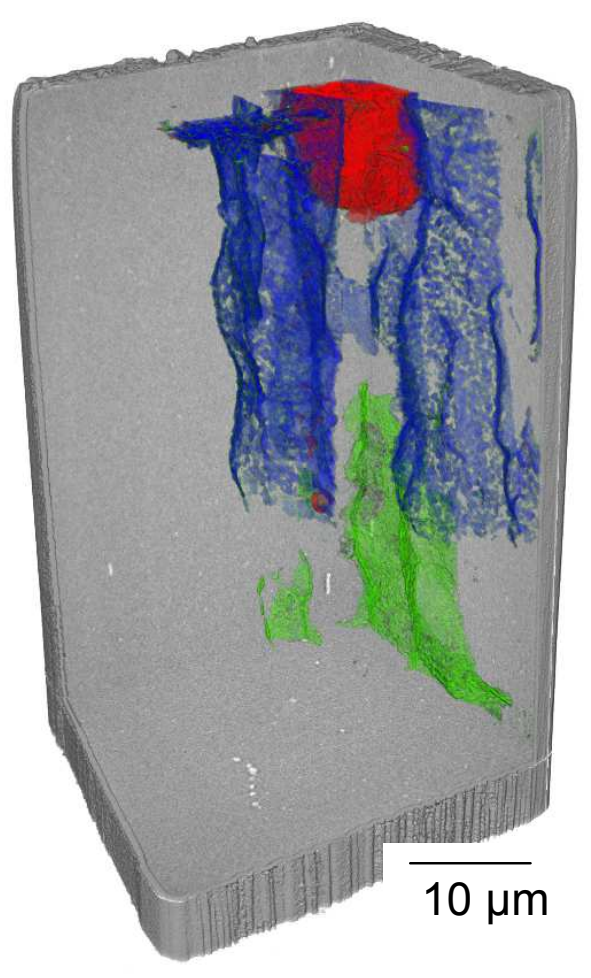

Fig. 7: A 3 D representation of the corrosion process of the alloy, with corrosion developed in 0.5 (blue), 2 (green), and $12 \mathrm{~h}$ (red) shown by the artificial colours. 


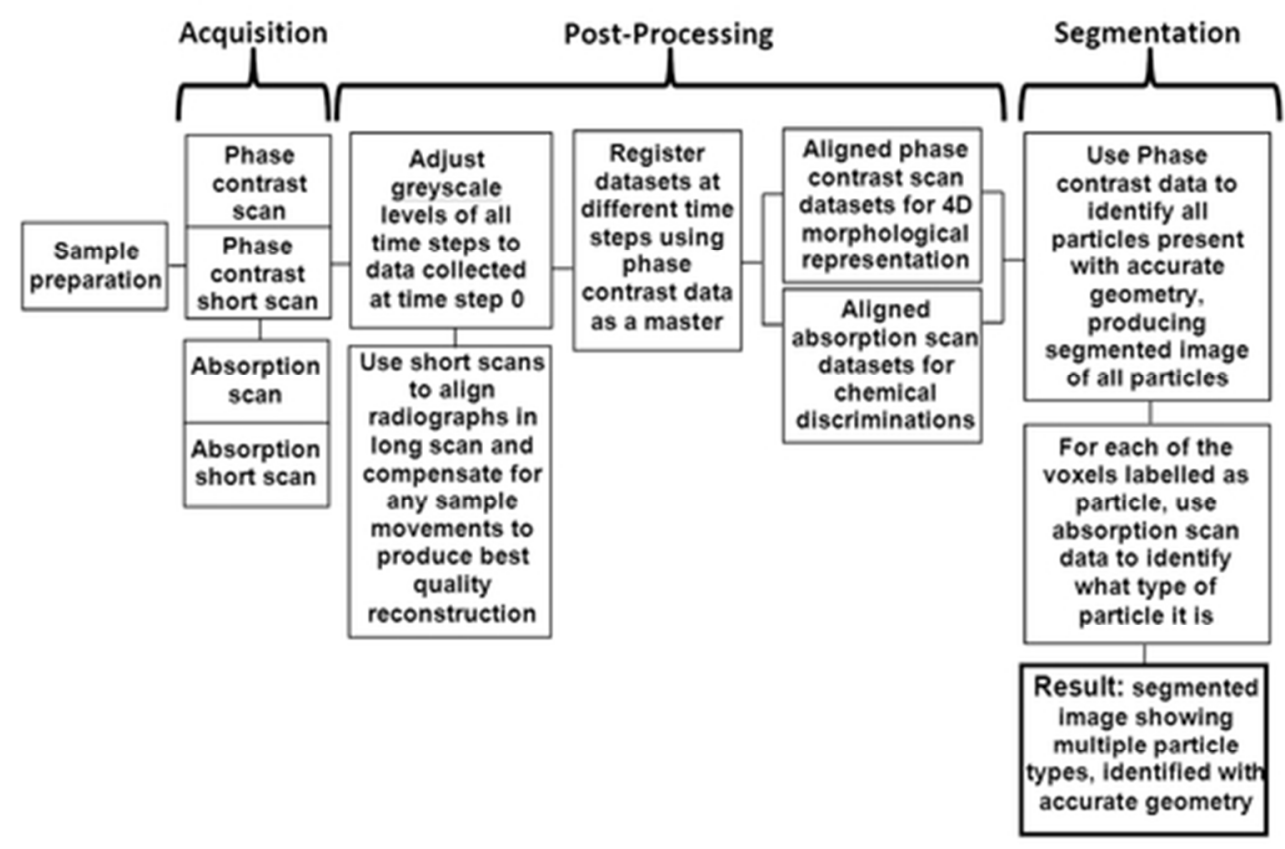

Fig. 1: A workflow diagram used for the time-lapse $\mathrm{x}$-ray nano-CT study of leaching inhibitors and corrosion of AA2099 aluminium alloy, revealing each steps in data acquisition and processing. 


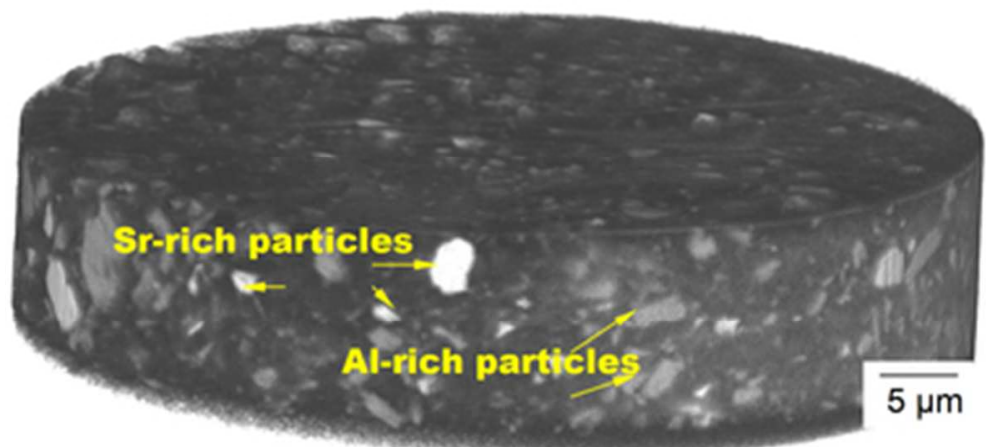

Fig. 2: A 3D x-ray nano-CT absorption image of the SAPP pigmented primer coating (14 micron thick on average).

$33 \times 15 \mathrm{~mm}(300 \times 300$ DPI $)$ 


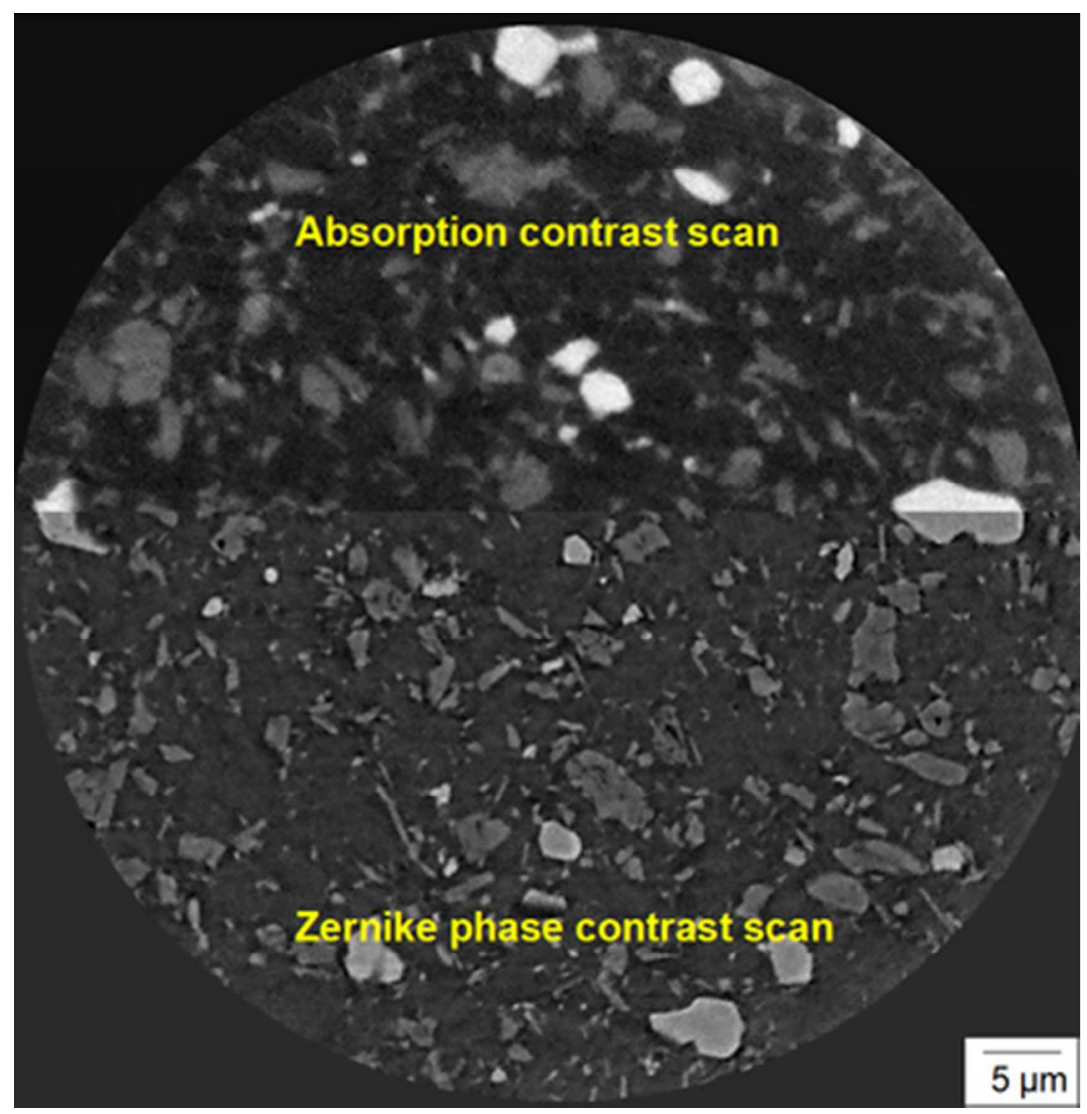

Fig. 3: A comparison between absorption (top part) and Zernike phase contrast scans (bottom part), which shows that the Zernike phase contrast scan reveals the morphology on greater detail while the absorption contrast reveals significant composition information, i.e. atomic contrast from different elements in the inhibitor.

$34 \times 35 \mathrm{~mm}(300 \times 300 \mathrm{DPI})$ 


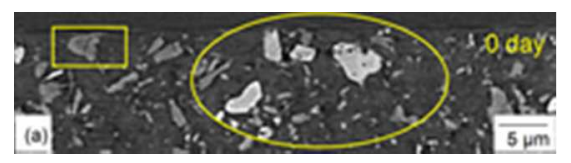

Fig. 4: The evolution of the organic primer coating pigmented with SAPP corrosion inhibitor over the course of the immersive treatment days in sodium chloride solution: (a) before immersion; (b) after immersion for 1 day; (c) after immersion for 7 days; (d) after immersion for 14 days. The oval region highlights the dissolution of strontium-rich particles while the square region highlighted the activity of aluminium-rich particles.

$17 \times 4 \mathrm{~mm}(300 \times 300 \mathrm{DPI})$ 


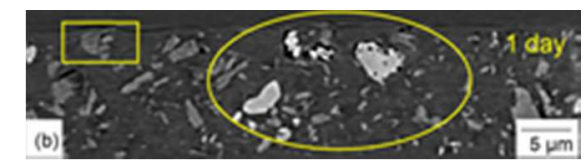

Fig. 4: The evolution of the organic primer coating pigmented with SAPP corrosion inhibitor over the course of the immersive treatment days in sodium chloride solution: (a) before immersion; (b) after immersion for 1 day; (c) after immersion for 7 days; (d) after immersion for 14 days. The oval region highlights the dissolution of strontium-rich particles while the square region highlighted the activity of aluminium-rich particles.

$18 \times 4 \mathrm{~mm}(300 \times 300 \mathrm{DPI})$ 


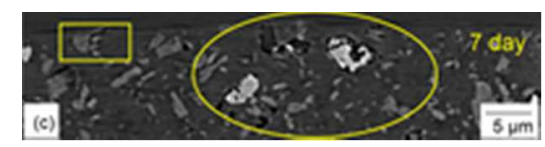

Fig. 4: The evolution of the organic primer coating pigmented with SAPP corrosion inhibitor over the course of the immersive treatment days in sodium chloride solution: (a) before immersion; (b) after immersion for 1 day; (c) after immersion for 7 days; (d) after immersion for 14 days. The oval region highlights the dissolution of strontium-rich particles while the square region highlighted the activity of aluminium-rich particles.

$16 \times 4 \mathrm{~mm}(300 \times 300 \mathrm{DPI})$ 


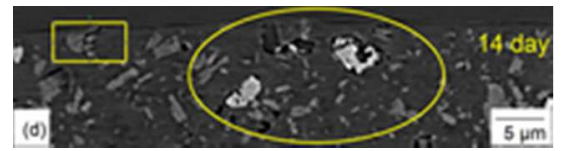

Fig. 4: The evolution of the organic primer coating pigmented with SAPP corrosion inhibitor over the course of the immersive treatment days in sodium chloride solution: (a) before immersion; (b) after immersion for 1 day; (c) after immersion for 7 days; (d) after immersion for 14 days. The oval region highlights the dissolution of strontium-rich particles while the square region highlighted the activity of aluminium-rich particles.

$17 \times 4 \mathrm{~mm}(300 \times 300 \mathrm{DPI})$ 


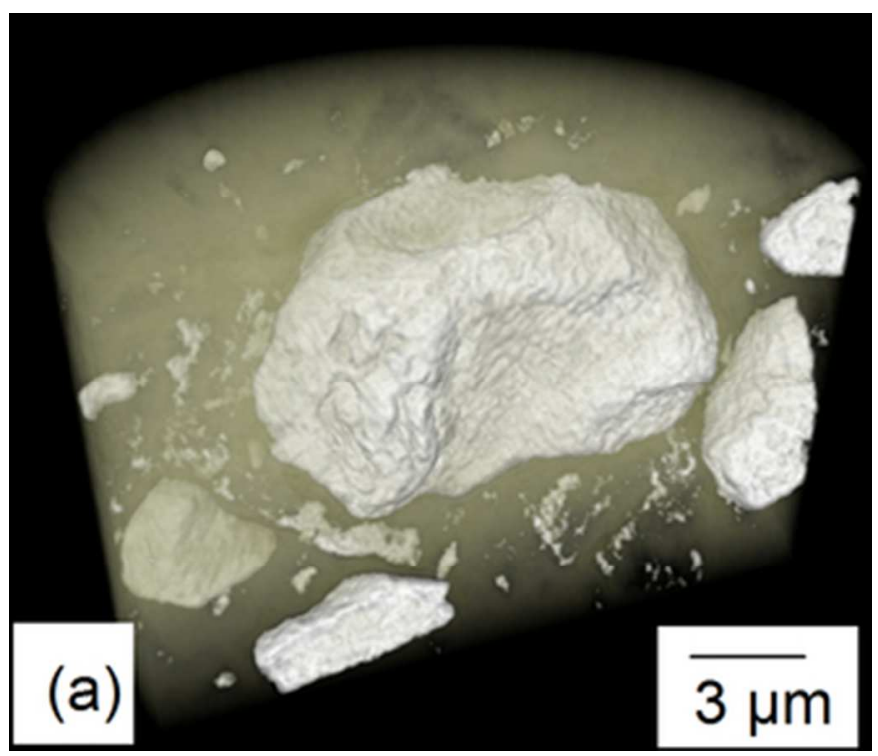

Fig. 5: A 3D representation of the dissolution of SAPP particles in the primer coating after immersion in 0.6 $\mathrm{M} \mathrm{NaCl}$ for the testing period: (a) before immersion; (b) after immersion for 1 day; (c) after immersion for 7 days; (d) after immersion for 14 days.

$28 \times 23 \mathrm{~mm}(300 \times 300$ DPI $)$ 


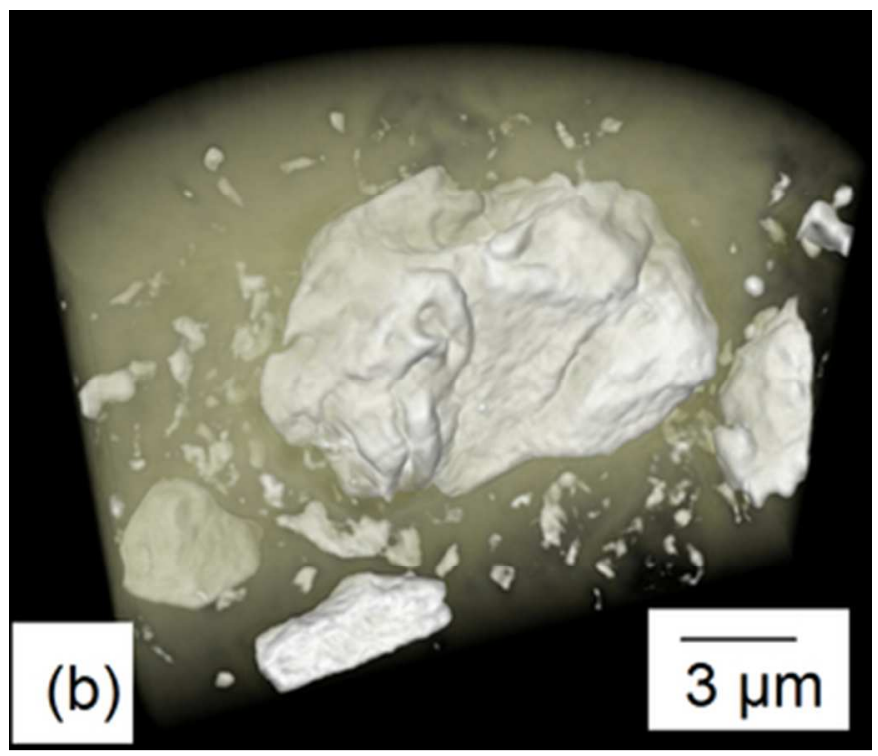

Fig. 5: A 3D representation of the dissolution of SAPP particles in the primer coating after immersion in 0.6 $\mathrm{M} \mathrm{NaCl}$ for the testing period: (a) before immersion; (b) after immersion for 1 day; (c) after immersion for 7 days; (d) after immersion for 14 days.

$28 \times 24 \mathrm{~mm}(300 \times 300$ DPI $)$ 


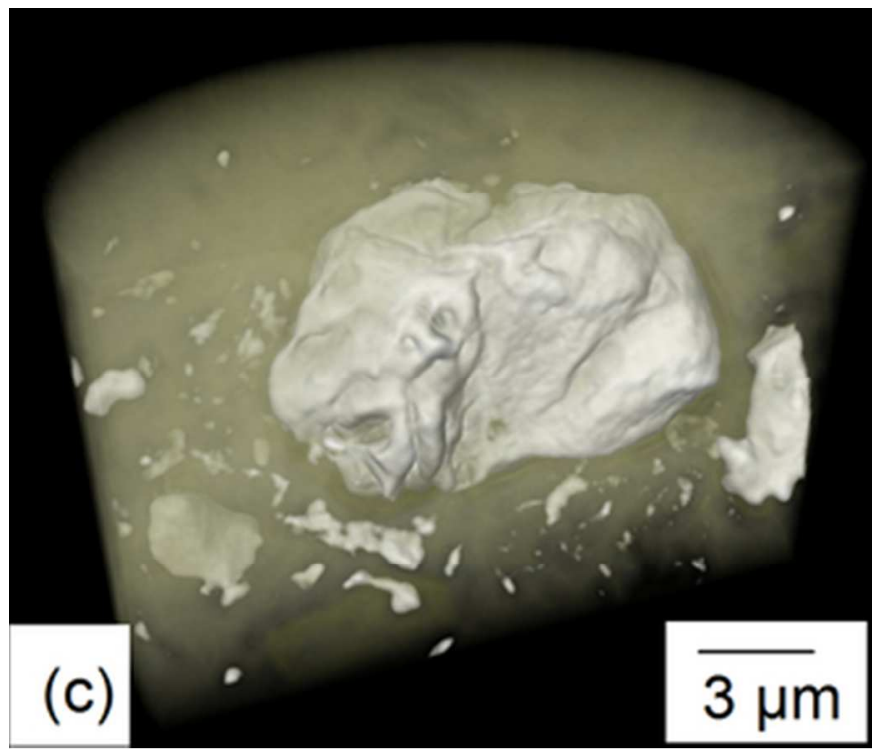

Fig. 5: A 3D representation of the dissolution of SAPP particles in the primer coating after immersion in 0.6 $\mathrm{M} \mathrm{NaCl}$ for the testing period: (a) before immersion; (b) after immersion for 1 day; (c) after immersion for 7 days; (d) after immersion for 14 days.

$28 \times 24 \mathrm{~mm}(300 \times 300$ DPI $)$ 


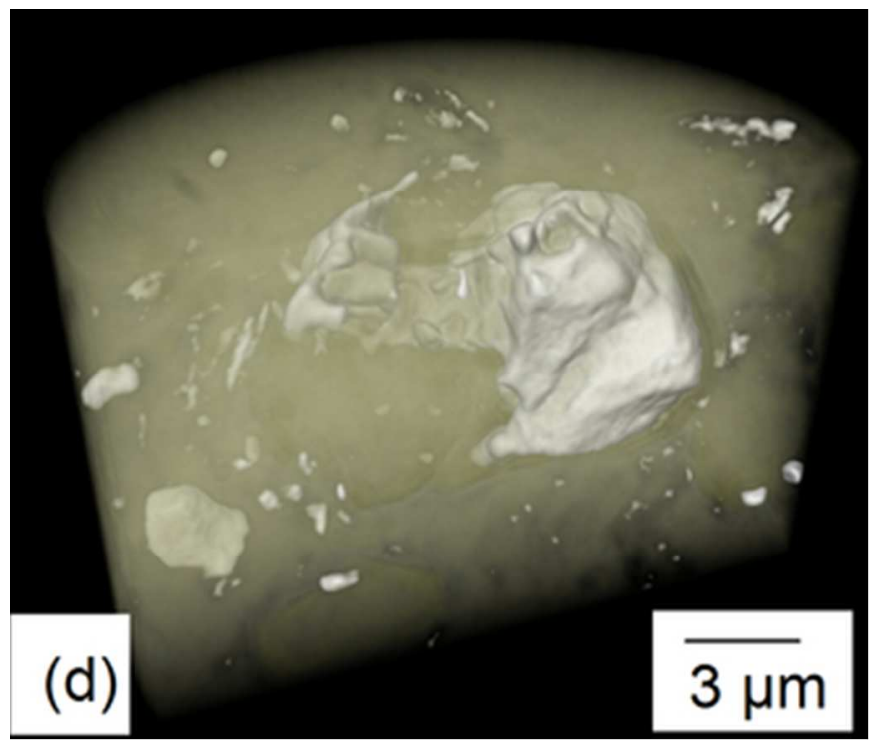

Fig. 5: A 3D representation of the dissolution of SAPP particles in the primer coating after immersion in 0.6 $\mathrm{M} \mathrm{NaCl}$ for the testing period: (a) before immersion; (b) after immersion for 1 day; (c) after immersion for 7 days; (d) after immersion for 14 days.

$27 \times 23 \mathrm{~mm}(300 \times 300$ DPI $)$ 


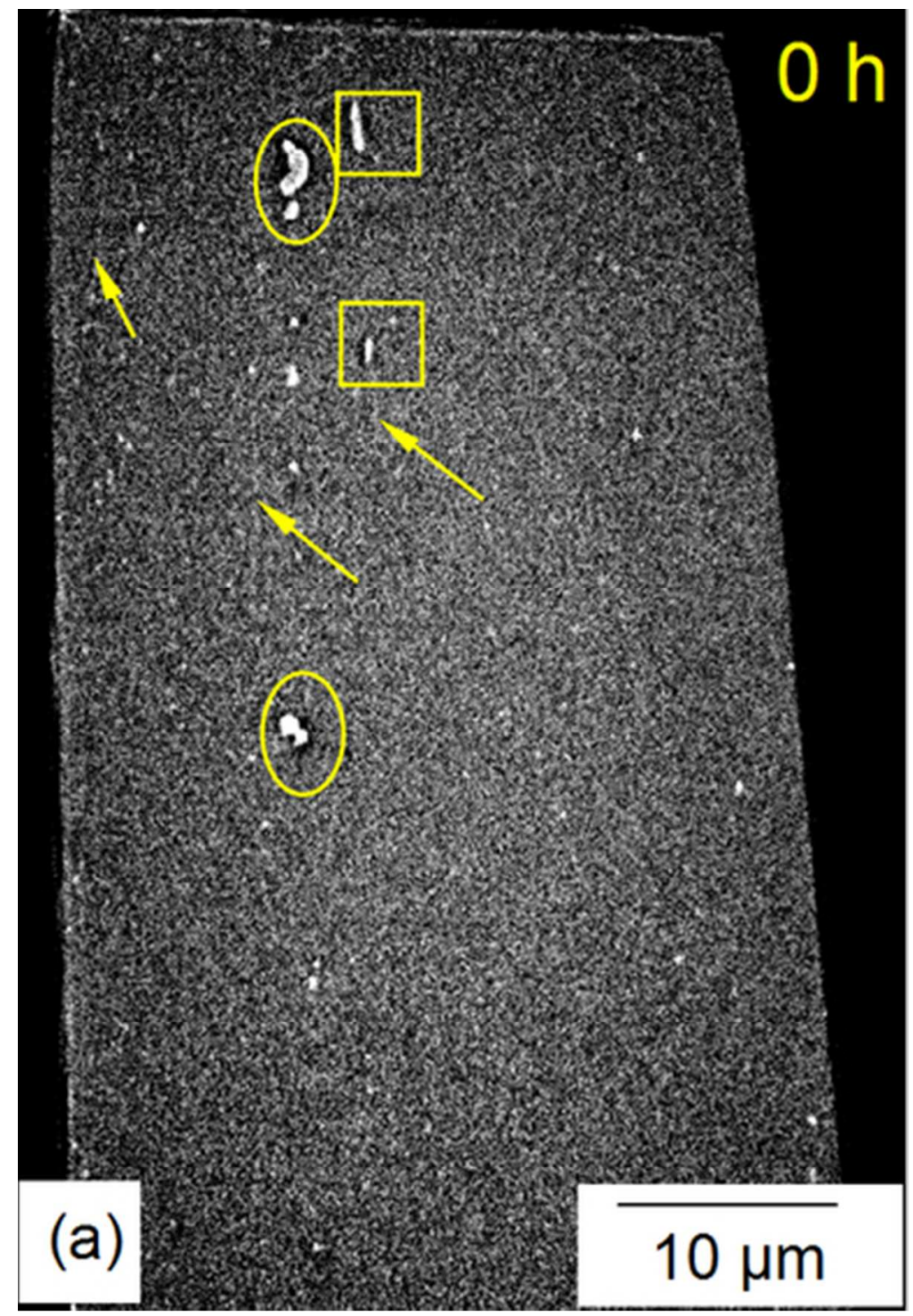

Fig. 6: Virtual cross-sections of Zernike phase contrast tomographic images of the alloy before (a) and after immersion in sodium chloride solution for $0.5 \mathrm{~h}$ (b), $2 \mathrm{~h}$ (c) and $12 \mathrm{~h}$ (d). 


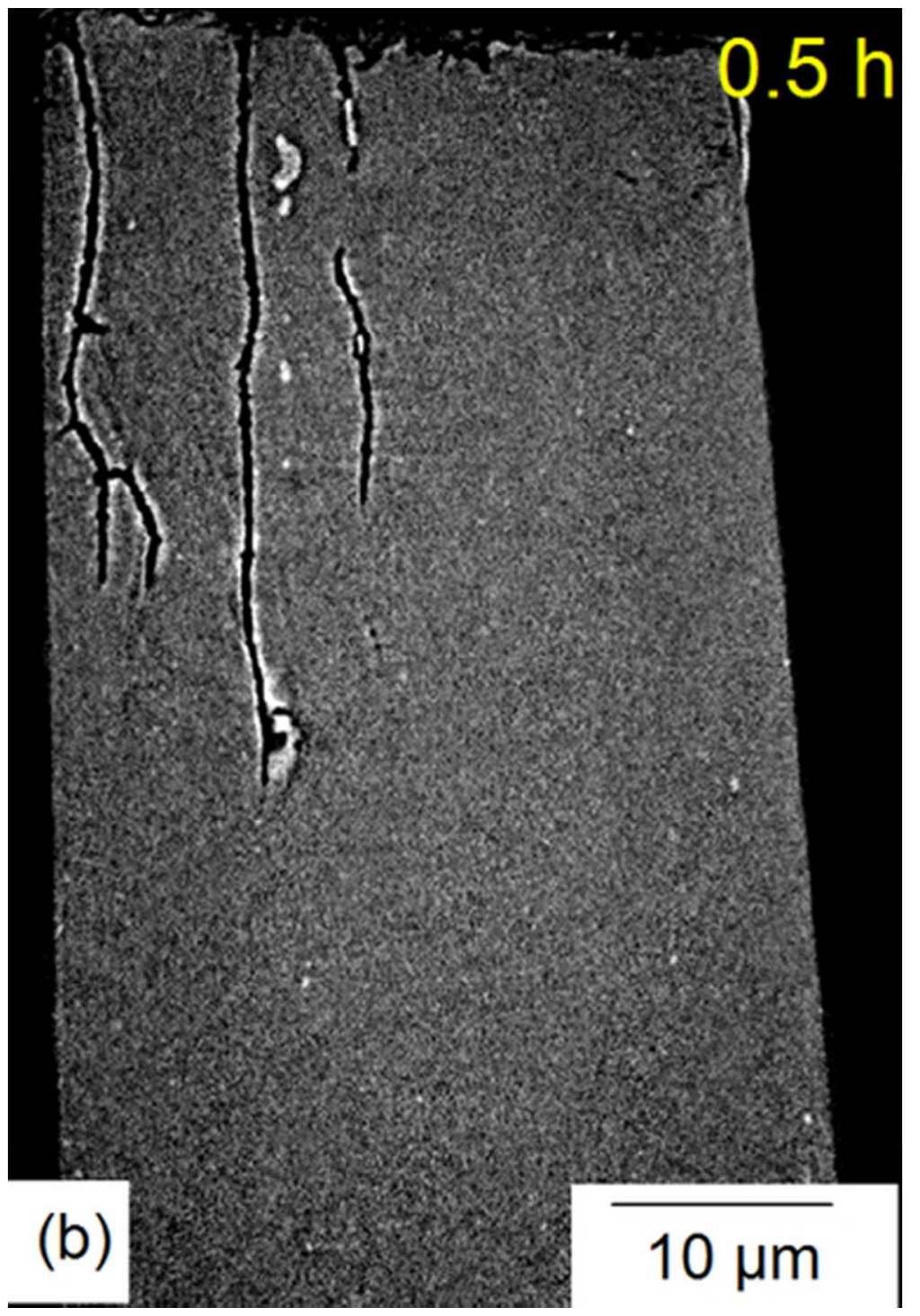

Fig. 6: Virtual cross-sections of Zernike phase contrast tomographic images of the alloy before (a) and after immersion in sodium chloride solution for $0.5 \mathrm{~h}(\mathrm{~b}), 2 \mathrm{~h}$ (c) and $12 \mathrm{~h}$ (d). 


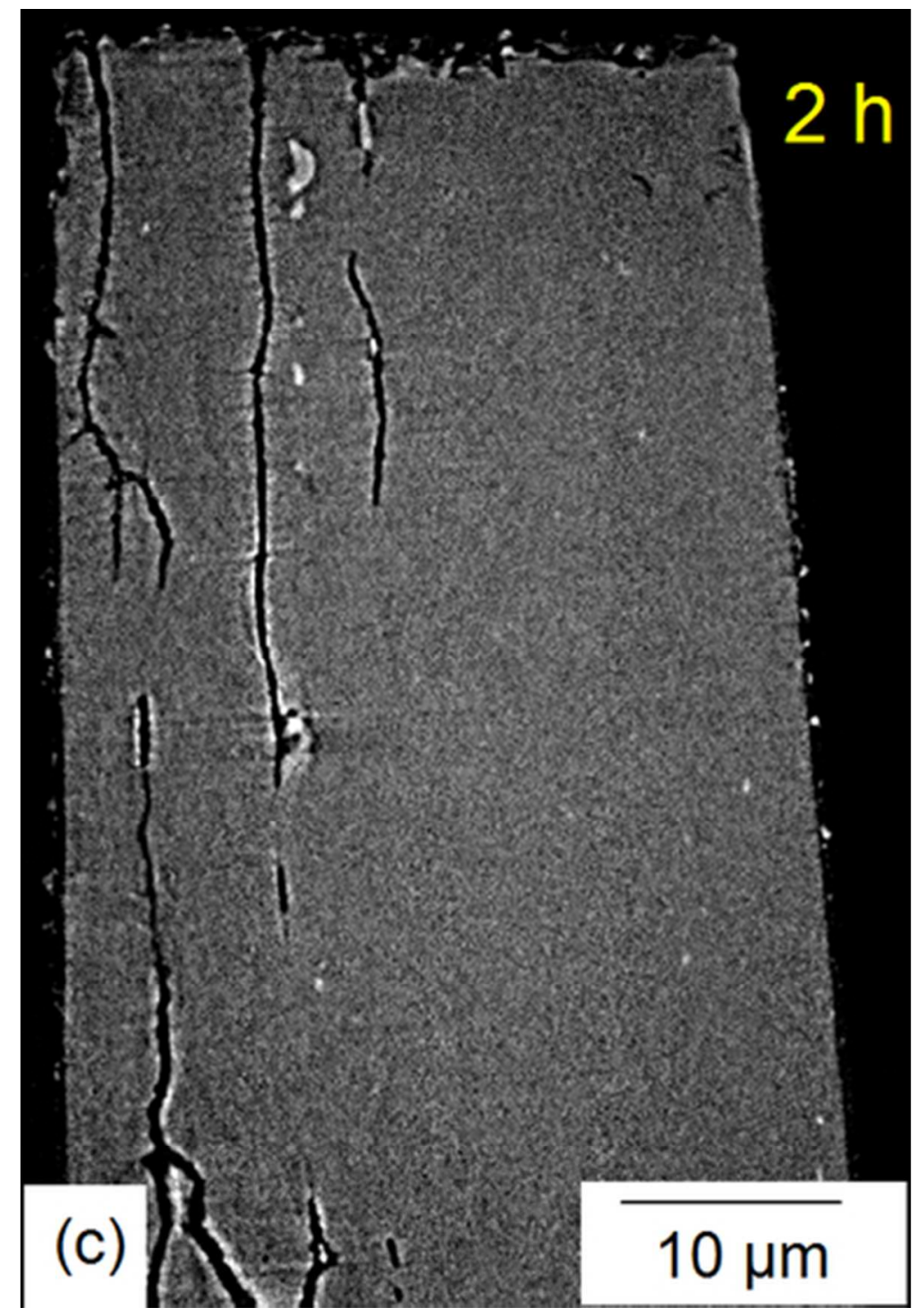

Fig. 6: Virtual cross-sections of Zernike phase contrast tomographic images of the alloy before (a) and after immersion in sodium chloride solution for $0.5 \mathrm{~h}$ (b), $2 \mathrm{~h}$ (c) and $12 \mathrm{~h}$ (d). 


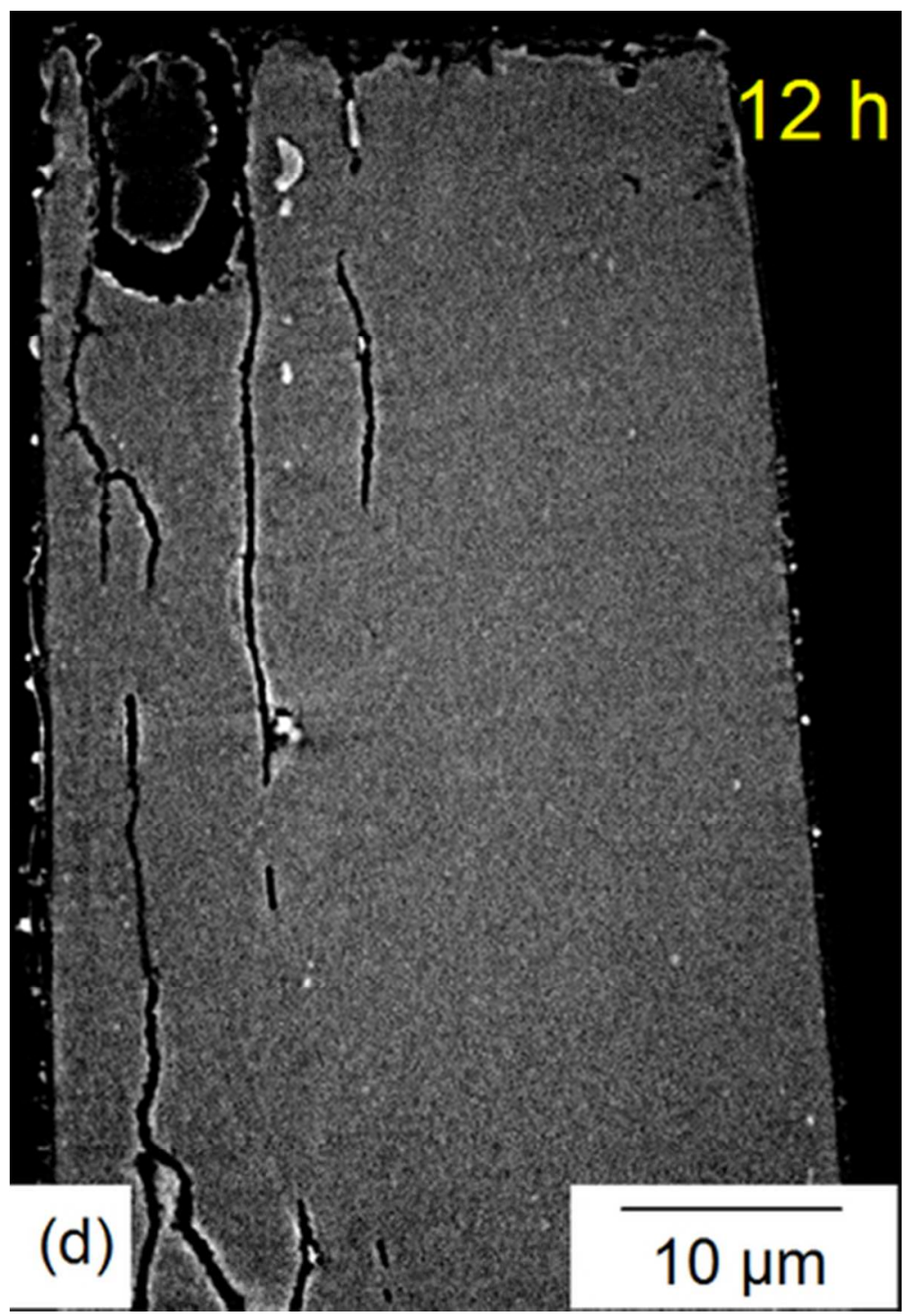

Fig. 6: Virtual cross-sections of Zernike phase contrast tomographic images of the alloy before (a) and after immersion in sodium chloride solution for $0.5 \mathrm{~h}$ (b), $2 \mathrm{~h}$ (c) and $12 \mathrm{~h} \mathrm{(d)}$. 


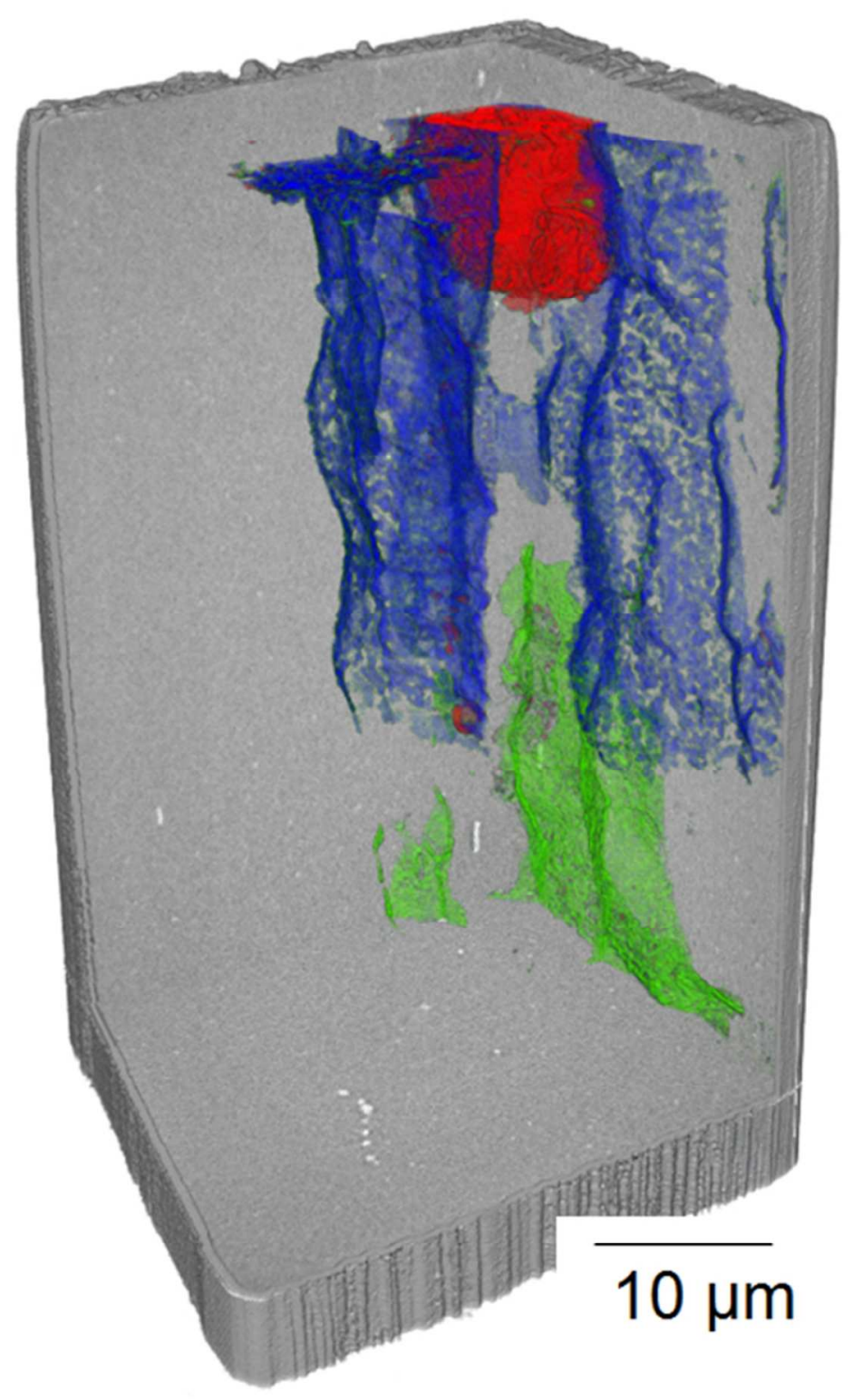

Fig. 7: A 3 D representation of the corrosion process of the alloy, with corrosion developed in 0.5 (blue), 2 (green), and $12 \mathrm{~h}$ (red) shown by the artificial colours.

$25 \times 37 \mathrm{~mm}(600 \times 600 \mathrm{DPI})$ 